\title{
Global Versus Local Casimir Effect
}

\author{
Andrzej Herdegen and Mariusz Stopa
}

\begin{abstract}
This paper continues the investigation of the Casimir effect with the use of the algebraic formulation of quantum field theory in the initial value setting. Basing on earlier papers by one of us (AH), we approximate the Dirichlet and Neumann boundary conditions by simple interaction models whose nonlocality in physical space is under strict control, but which at the same time are admissible from the point of view of algebraic restrictions imposed on models in the context of Casimir backreaction. The geometrical setting is that of the original parallel plates. By scaling our models and taking appropriate limit, we approach the sharp boundary conditions in the limit. The global force is analyzed in that limit. One finds in Neumann case that although the sharp boundary interaction is recovered in the norm resolvent sense for each model considered, the total force per area depends substantially on its choice and diverges in the sharp boundary conditions limit. On the other hand the local energy density outside the interaction region, which in the limit includes any compact set outside the strict position of the plates, has a universal limit corresponding to sharp conditions. This is what one should expect in general, and the lack of this discrepancy in Dirichlet case is rather accidental. Our discussion pins down its precise origin: the difference in the order in which scaling limit and integration over the whole space is carried out.
\end{abstract}

\section{Introduction and the Main Idea}

The most natural setting for the consideration of the Casimir effect is the algebraic approach. This approach allows a mathematically rigorous analysis of the effect and gives a clear understanding of the sources of the difficulties one encounters in more traditional treatments. In application to quantum fields this analysis rests, in broad terms, on the following cornerstones.

(i) A quantum relativistic theory is defined by an algebra of observables, in simple cases defined directly by 'fields' (scalar, electromagnetic).

(ii) Each particular physical system obeying this theory is described by a Hilbert space representation of this algebra. Inequivalent representations 
refer to physically non-comparable systems or idealizations (such as a local isolated system and a thermodynamic limit system).

(iii) The change of external conditions under which a quantum system is placed leads to a change of the state of the system.

For the calculation of the global Casimir-type effects, as the backreaction force, one needs models which respect the above three constituents of a quantum theory. Thus the model of a quantum field should be based on one definite algebra, and the interaction with the external conditions should not lead to a change of its representation. If this condition is fulfilled then the Casimir force results from the change in the expectation value of one and the same energy observable, as defined by free field, as the state changes with changing external conditions (such as position of macroscopic bodies).

This analysis has been conducted at length by one of us in $[1,2]$, where also clear cut criterions for the admissibility of external interaction models for a class of systems were formulated. In application to the free quantum scalar field these amount to the following. Let $\phi(x)$ be a scalar field and denote by $h^{2}$ the standard self-adjoint extension in $L^{2}\left(\mathbb{R}^{3}, \mathrm{~d}^{3} x\right)$ of $-\Delta$. The free field dynamics is then

$$
\left(\partial_{t}^{2}+h^{2}\right) \phi(t, \vec{x})=0 .
$$

Suppose now that one introduces to the system external macroscopic bodies, such as conducting plates in the original Casimir system, which change the dynamics of the field. Let $a$ denote free parameters of these bodies, such as separation of the plates, and let the modified dynamics (for fixed $a$ ) be given by

$$
\left(\partial_{t}^{2}+h_{a}^{2}\right) \phi(t, \vec{x})=0,
$$

where $h_{a}^{2}$ is a positive self-adjoint operator. Now, the two settings can be described by one choice of observables algebra, and in common representation of this algebra if, and only if, $h_{a}^{-1 / 2}\left(h_{a}-h\right) h^{-1 / 2}$ is a Hilbert-Schmidt operator in $L^{2}\left(\mathbb{R}^{3}, \mathrm{~d}^{3} x\right)$, that is

$$
\operatorname{Tr}\left[h^{-1 / 2}\left(h_{a}-h\right) h_{a}^{-1}\left(h_{a}-h\right) h^{-1 / 2}\right]<\infty .
$$

Suppose this condition holds and let the algebra of the field be represented in some Hilbert space $\mathcal{H}$. Let further $H$ be the energy operator as defined by free field dynamics, and let $\Omega_{a}$ be the minimal energy state vector as defined by the modified dynamics (2). The Casimir energy is then given by

$$
\mathcal{E}_{a}=\left(\Omega_{a}, H \Omega_{a}\right)=\frac{1}{4} \operatorname{Tr}\left[\left(h_{a}-h\right) h_{a}^{-1}\left(h_{a}-h\right)\right] .
$$

That this energy be finite is another condition on the model of $h_{a}$, and only if both conditions are satisfied the Casimir problem has a finite solution and the Casimir force is then

$$
\mathcal{F}_{a}=-\frac{\mathrm{d} \mathcal{E}_{a}}{\mathrm{~d} a}
$$


These admissibility conditions say, roughly, that the modified dynamics $h_{a}^{2}$ cannot differ much from the free dynamics $h^{2}$. Introducing sharp boundary conditions, such as Dirichlet/Neumann conditions on plates, violates these demands. One faces therefore the problem of an appropriate approximation for the description of such plates.

We consider the simplest geometrical situation, the original Casimir problem of two infinite, parallel plane plates at a distance $a$ from each other. We assume that $z$-axis is perpendicular to the planes and the modification of dynamics affects this direction only, thus

$$
h^{2}=h_{z}^{2}+h_{\perp}^{2}, \quad h_{a}^{2}=h_{z a}^{2}+h_{\perp}^{2} .
$$

where $h_{\perp}^{2}$ is the free dynamics in the directions perpendicular to $z$-axis and $h_{z a}^{2}$ is a modification of $h_{z}^{2}=-\partial_{z}^{2}$ in $L^{2}(\mathbb{R}, \mathrm{d} z)$; we refer the reader for details to [2]. In this setting the conditions of finiteness of (3) and (4) cannot be expected to hold as they stand because of translation symmetry in the planes, and must be replaced by conditions 'per unit area' of the planes. It has been shown in [2] that this amounts to

$$
\begin{aligned}
& \operatorname{Tr}\left[\left(h_{z a}-h_{z}\right)^{2}\right]<\infty, \\
& \operatorname{Tr}\left[\left(h_{z a}-h_{z}\right) h_{z}\left(h_{z a}-h_{z}\right)\right]<\infty,
\end{aligned}
$$

where the trace refers to the Hilbert space $L^{2}(\mathbb{R}, \mathrm{d} z)$. If these conditions are satisfied, the energy per unit area is finite and reads

$$
\varepsilon_{a}=\frac{1}{24 \pi} \operatorname{Tr}\left[\left(h_{z a}-h_{z}\right)\left(2 h_{z}+h_{z a}\right)\left(h_{z a}-h_{z}\right)\right] .
$$

A class of models for $h_{z a}^{2}$ imitating the boundary conditions, but consistent with the above demands, was considered in [2]. The idea was to take $h_{z a}^{2}=($ function of $)\left(h_{z}, h_{z a}^{B}\right)$, where $\left(h_{z a}^{B}\right)^{2}$ is $-\partial_{z}^{2}$ with boundary conditions at $z= \pm a / 2$. The choice of functions assured that for small spectral values of $h_{z}^{2}$ and $\left(h_{z a}^{B}\right)^{2}$ the models reproduced the sharp boundaries, while for large spectral values tended to free dynamics. Moreover, one could introduce a scaling parameter $\mu$ such that for $\mu \rightarrow \infty$ the models approached the sharp boundaries in the whole spectrum. For the Casimir energy per area in the rescaled models $\varepsilon_{a}^{\lambda}$ (we prefer to work with $\lambda=1 / \mu$ here) one then found

$$
\varepsilon_{a}^{\lambda}=\frac{\varepsilon_{\infty}}{\lambda^{3}}+\frac{c}{\lambda a^{2}}-\frac{\pi^{2}}{1440 a^{3}}+(\text { terms } \rightarrow 0 \text { for } \lambda \rightarrow 0),
$$

where $\varepsilon_{\infty}$ and $c$ are constants, $c=0$ in Dirichlet case, but $c \neq 0$ in Neumann case. It was also shown that the direct sum of the two models describes the setting of the electromagnetic field between conducting plates. Thus the Casimir force per unit area is then

$$
-\frac{\mathrm{d}\left[\varepsilon_{a}^{\lambda}(D)+\varepsilon_{a}^{\lambda}(N)\right]}{\mathrm{d} a}=\frac{2 c}{\lambda a^{3}}-\frac{\pi^{2}}{240 a^{4}}+(\text { terms } \rightarrow 0 \text { for } \lambda \rightarrow 0) .
$$

The second term reproduces the well-known Casimir's formula, but the first term is model-dependent and dominates for large $a$. Moreover, in typical situations there is $c>0$ and the force becomes repulsive for large $a$. 
In the present paper we want to find out whether these results will be confirmed in another class of models, constructed in a wholly different way. Rather than manipulate spectral properties directly, now we want to approximate interaction with the plates directly in the physical space. It is easy to see that strictly local potential interaction of the form $\left[h_{z a}^{2} \psi\right](z)=-\partial_{z}^{2} \psi(z)+V(z) \psi(z)$ violates our conditions. Therefore we replace $V$ by a slightly nonlocal integral quasi-potential $[V \psi](z)=\int V\left(z, z^{\prime}\right) \psi\left(z^{\prime}\right) \mathrm{d} z^{\prime}$ with the kernel $V\left(z, z^{\prime}\right)$ concentrated around the position of the plates. We show that with an appropriately defined scaling of some simple kernels of this kind one can reproduce sharp boundary conditions on the plates in the limit. The Casimir energy can again be calculated and for a class of models the result (8) is confirmed. However, in general the Neumann case proves to be even more singular here than in the models considered in [2] and the universal term could be disturbed. Nonlocal quasi-potentials has been considered in the Casimir context by other authors before, but in different formalisms and with rather different motivations (see e.g. $[3,4])$.

The present choice of models makes also possible a local analysis of the local energy density. We show that outside the interaction region the density tends in the scaling limit to a well defined universal form corresponding to sharp boundary conditions. The present mathematically rigorous setting allows the comparison and better understanding of the local-global relation. The model-dependent and divergent (in the limit) contributions to the global force are due to the interaction region. We discuss this point more fully in the Discussion section.

For a more extensive discussion of the background of the present paper, as well as for more extensive literature we refer the reader to $[1,2]$. We define our models in Sect. 2. Appropriate scaling of these models is shown to reproduce the sharp boundary conditions in Sect. 3. Spectral properties of the models are discussed in Sect. 4 and the admissibility of the models in the sense mentioned above is proved in Sect. 5. It is shown in Sect. 6 that the Casimir energy of the scaled models is obtained by the expansion of the formula for energy in inverse powers of $a$, and this expansion (up to a significant order) is obtained in Sect. 7. For comparison, in Sect. 8 we obtain local results and their scaling limit. The discussion occupies Sect. 9. More technical points of our derivations are shifted to Appendices.

\section{The Models}

We postulate for our analysis the following quasi-potentials

$$
V=\sigma\left(\left|U_{b} g\right\rangle\left\langle U_{b} g|+| U_{-b} g\right\rangle\left\langle U_{-b} g\right|\right), \quad b=a / 2>0, \quad \sigma= \pm 1,
$$

where $U$ is the translation operator and $\sigma=1,-1$ corresponds to Dirichlet (D) and Neumann (N) conditions respectively. These conditions will be achieved in the two cases by an appropriate scaling limit to be defined below. In all what follows one should keep in mind that unless stated otherwise we treat parallelly both cases, but the dependence of quantities on $\sigma$ is suppressed. 
In position representation the quasi-potential is an integral operator $(V \psi)(z)=\int V\left(z, z^{\prime}\right) \psi\left(z^{\prime}\right) \mathrm{d} z^{\prime}$ with the kernel

$$
V\left(z, z^{\prime}\right)=\sigma\left[g(z-b) \overline{g\left(z^{\prime}-b\right)}+g(z+b) \overline{g\left(z^{\prime}+b\right)}\right] .
$$

For the functions $g$ we assume that

$$
g(z)= \begin{cases}f(z) & \text { if } \sigma=1 \\ -i \frac{\mathrm{d}}{\mathrm{d} z} f(z) & \text { if } \sigma=-1\end{cases}
$$

where $f$ is a complex, compactly supported smooth function, with the following properties

$$
\begin{aligned}
f(-z) & =f(z), \quad \operatorname{supp} f \subseteq\langle-R, R\rangle, R<b, \quad \widehat{f}(0) \neq 0, \\
\|f\| & =1, \quad(\mathrm{~N})
\end{aligned}
$$

where $\widehat{f}$ is the Fourier-transformed function

$$
\widehat{f}(p)=\frac{1}{\sqrt{2 \pi}} \int f(z) \mathrm{e}^{-i p z} \mathrm{~d} z,
$$

and the last property is assumed only in the Neumann case. The first condition reflects the symmetry of each of the plates, the second says that the nonlocalities of the two interaction centers at $z= \pm b$ do not overlap, and the third and fourth are technical.

We denote by $h_{z}$ and $h_{z a}$ the self-adjoint, non-negative square roots of the operators

$$
h_{z}^{2}=-(\mathrm{d} / \mathrm{d} z)^{2}, \quad h_{z a}^{2}=h_{z}^{2}+V,
$$

respectively. Operator $h_{z}^{2}$ is the standard one-dimensional Laplace operator (with opposite sign), while $h_{z a}^{2}$ is its Kato-Rellich perturbation, with unchanged domain, as $V$ is bounded. The (strict) positivity of $h_{z a}^{2}$ in the Dirichlet case is obvious, while in the Neumann case one has by (10) that for all $\psi$ in the domain of $h_{z}^{2}$ there is

$$
\left(\psi, h_{z a}^{2} \psi\right)=\left\|\psi^{\prime}\right\|^{2}-\left|\left(U_{+b} f, \psi^{\prime}\right)\right|^{2}-\left|\left(U_{-b} f, \psi^{\prime}\right)\right|^{2},
$$

where $\psi^{\prime}(z)=\mathrm{d} \psi(z) / \mathrm{d} z$. The functions $U_{ \pm b} f$ do not overlap, and satisfy $\left\|U_{ \pm b} f\right\|=\|f\|$. Thus by (12) they form an orthonormal system, which implies $\left(\psi, h_{z a}^{2} \psi\right) \geq 0$ for each $\psi$. (Here a more strongly bounding condition $\|f\|<1$ would produce a strictly positive operator; this, however, would not lead to the recovery of Neumann condition in a limit to be defined below.)

For $w^{2} \in \mathbb{C}$ with $\mathfrak{I m} w^{2} \neq 0$ the resolvents denoted by

$$
G_{0}\left(w^{2}\right)=\left(w^{2}-h_{z}^{2}\right)^{-1}, \quad G\left(w^{2}\right)=\left(w^{2}-h_{z a}^{2}\right)^{-1},
$$

are bounded operators. In all what follows for given $w^{2}$ we fix $w$ by $\mathfrak{I m} w>0$. We also introduce the $T$-operator known from the stationary scattering theory

$$
T\left(w^{2}\right)=V+V G_{0}\left(w^{2}\right) T\left(w^{2}\right) .
$$


This equation may be explicitly solved for $T$ : making the Ansatz

$$
T\left(w^{2}\right)=\left(\begin{array}{ll}
\left|U_{+b} g\right\rangle & \left|U_{-b} g\right\rangle
\end{array}\right) \mathcal{T}\left(w^{2}\right)\left(\begin{array}{c}
\left\langle U_{+b} g\right| \\
\left\langle U_{-b} g\right|
\end{array}\right),
$$

with $\mathcal{T}\left(w^{2}\right)$ a numerical matrix, one easily finds

$$
\mathcal{T}\left(w^{2}\right)=\left(\begin{array}{ll}
\sigma-\left(g, G_{0}\left(w^{2}\right) g\right)-\left(U_{a} g, G_{0}\left(w^{2}\right) g\right) \\
-\left(U_{a} g, G_{0}\left(w^{2}\right) g\right) & \sigma-\left(g, G_{0}\left(w^{2}\right) g\right)
\end{array}\right)^{-1} .
$$

The resolvent may be then expressed by

$$
G\left(w^{2}\right)=G_{0}\left(w^{2}\right)+G_{0}\left(w^{2}\right) T\left(w^{2}\right) G_{0}\left(w^{2}\right) .
$$

In momentum representation, taking into account (10) and denoting

$$
\mathcal{F}_{p}=p^{\frac{1-\sigma}{2}} \widehat{f}(p) \quad\left(\mathrm{e}^{-i b p} \mathrm{e}^{+i b p}\right), \quad M_{p}=|\widehat{f}(p)|^{2},
$$

we have

$$
\begin{aligned}
\left\langle p\left|T\left(w^{2}\right)\right| q\right\rangle & =\mathcal{F}_{p} \mathcal{T}\left(w^{2}\right) \mathcal{F}_{q}^{\dagger}, \\
\left\langle p\left|G\left(w^{2}\right)-G_{0}\left(w^{2}\right)\right| q\right\rangle & =\frac{\mathcal{F}_{p}}{w^{2}-p^{2}} \mathcal{T}\left(w^{2}\right) \frac{\mathcal{F}_{q}^{\dagger}}{w^{2}-q^{2}},
\end{aligned}
$$

with elements in the matrix (17) given by

$$
\begin{aligned}
\sigma-\left(g, G_{0}\left(w^{2}\right) g\right) & =\sigma-\int \frac{p^{1-\sigma} M_{p}}{w^{2}-p^{2}} \mathrm{~d} p \\
-\left(U_{a} g, G_{0}\left(w^{2}\right) g\right) & =-\int \frac{\mathrm{e}^{i a p} p^{1-\sigma} M_{p}}{w^{2}-p^{2}} \mathrm{~d} p=i \pi w^{-\sigma} \mathrm{e}^{i a w} M_{w} .
\end{aligned}
$$

The integral in the last formula is calculated in the complex plane by residues, with the use of analyticity and asymptotic properties of $M_{w}$ discussed at the beginning of Appendix A.

\section{Reproduction of the Sharp Boundary Conditions}

We consider now a family of rescaled quasi-potentials $V_{\lambda}, \lambda \in(0,1)$, built as in (9), but with the use of rescaled functions $g_{\lambda}$ instead of $g$. We write the scaling in several equivalent forms:

$$
g_{\lambda}(z)=\lambda^{-\frac{3}{2}} g\left(\frac{z}{\lambda}\right), \quad f_{\lambda}(z)=\lambda^{-1-\frac{\sigma}{2}} f\left(\frac{z}{\lambda}\right), \quad \widehat{f}_{\lambda}(p)=\lambda^{-\frac{\sigma}{2}} \widehat{f}(\lambda p),
$$

and note also that $M_{p, \lambda}=\lambda^{-\sigma} M_{p}$. The rescaled potentials give rise to the corresponding operators $h_{z a, \lambda}$. All quantities referring to these operators acquire the subscript $\lambda$.

Let $h_{z a}^{B}$ be the self-adjoint, positive square root of the operator

$$
\left[h_{z a}^{B}\right]^{2}=\left[-\frac{\mathrm{d}^{2}}{\mathrm{~d} z^{2}}\right]_{\text {boundary conditions }}
$$


with standard domains in $L^{2}(\mathbb{R})$, Dirichlet/Neumann (for $\sigma=+1 /-1$ ) conditions in $z= \pm b$, and denote by $G^{B}\left(w^{2}\right)$ the resolvent of $\left[h_{z a}^{B}\right]^{2}$. Our objective in this section is to show the limiting property

$$
\lim _{\lambda \rightarrow 0^{+}}\left\|G_{\lambda}\left(w^{2}\right)-G^{B}\left(w^{2}\right)\right\|_{\mathrm{HS}}=0,
$$

where the Hilbert-Schmidt norm is $\|A\|_{\mathrm{HS}}^{2}=\operatorname{Tr}\left(A^{*} A\right) \geq\|A\|^{2}$. From this relation it follows then the norm convergence

$$
\lim _{\lambda \rightarrow 0^{+}}\left\|F\left(h_{z a, \lambda}\right)-F\left(h_{z a}^{B}\right)\right\|=0
$$

for each continuous and vanishing in infinity complex function $F$ on $\mathbb{R}$, and the strong convergence

$$
\lim _{\lambda \rightarrow 0^{+}}\left\|\left[F\left(h_{z a, \lambda}\right)-F\left(h_{z a}^{B}\right)\right] \psi\right\|=0,
$$

for each bounded continuous function $F$ and vector $\psi \in L^{2}$.

It is clear from the form of Eq. (21) that $G\left(w^{2}\right)-G_{0}\left(w^{2}\right)$ and its scaled version $G_{\lambda}\left(w^{2}\right)-G_{0}\left(w^{2}\right)$ are finite rank, hence Hilbert-Schmidt, operators. Thus it is sufficient to calculate the strong $-L^{2}\left(\mathbb{R}^{2}, \mathrm{~d} p \mathrm{~d} q\right)$ limit for $\lambda \rightarrow 0^{+}$of the integral kernel $\left\langle p\left|G_{\lambda}\left(w^{2}\right)-G_{0}\left(w^{2}\right)\right| q\right\rangle$. First we consider the numerical matrix $\mathcal{T}_{\lambda}$, and for later use we also look at higher orders in $\lambda$. We observe that

$$
\frac{1}{\lambda} \int \frac{M_{\lambda p}-M_{0}}{w^{2}-p^{2}} \mathrm{~d} p=I_{0}+O(\lambda)
$$

with

$$
I_{0}=\int \frac{M_{0}-M_{p}}{p^{2}} \mathrm{~d} p
$$

This is shown by writing the difference of (26) and (27) as

$$
\lambda w \int \frac{M_{u}-M_{0}}{u^{2}} \frac{\lambda w}{(\lambda w)^{2}-u^{2}} \mathrm{~d} u
$$

and Fourier-transforming the integral as a scalar product of two $L^{2}$-functions. This integral is shown in this way to be bounded by a constant. Using (22), (23), (26) and the assumption (12) (in Neumann case), we find

$$
\begin{aligned}
& \sigma-\left(g_{\lambda}, G_{0}\left(w^{2}\right) g_{\lambda}\right)=i \pi(\lambda w)^{-\sigma} M_{0}+(\lambda w)^{1-\sigma}\left(\frac{1+\sigma}{2}-I_{0}\right)+O\left(\lambda^{2-\sigma}\right), \\
& -\left(U_{a} g_{\lambda}, G_{0}\left(w^{2}\right) g_{\lambda}\right)=i \pi(\lambda w)^{-\sigma} \mathrm{e}^{i a w} M_{0}+O\left(\lambda^{2-\sigma}\right) .
\end{aligned}
$$

From these we get

$$
\begin{aligned}
& \mathcal{T}_{\lambda}\left(w^{2}\right)=\frac{-i(\lambda w)^{\sigma}}{\pi M_{0}\left(1-\mathrm{e}^{2 i a w}\right)}\left(\begin{array}{cc}
1 & -\mathrm{e}^{i a w} \\
\text { hboxe } & 1
\end{array}\right) \\
& +(\lambda w)^{1+\sigma}\left(\frac{1+\sigma}{2}-I_{0}\right) \times\{\text { matrix independent of } \lambda\}+O\left(\lambda^{2+\sigma}\right) .
\end{aligned}
$$

Next, we observe that (this is shown in Appendix A)

$$
\left\|\frac{\lambda^{\frac{\sigma}{2}} \widehat{f}_{\lambda}(p) p^{\frac{1-\sigma}{2}}}{w^{2}-p^{2}}-\frac{\widehat{f}(0) p^{\frac{1-\sigma}{2}}}{w^{2}-p^{2}}\right\| \leq\left\{\begin{array}{l}
\operatorname{const}(w) \lambda^{\frac{3}{2}}, \\
\operatorname{const}(w) \lambda^{\frac{1}{2}}
\end{array}\right.
$$


and in addition, for Neumann case

$$
\left\|\frac{\lambda^{-\frac{1}{2}} \widehat{f}_{\lambda}(p)}{w^{2}-p^{2}}-\frac{\widehat{f}(0)}{w^{2}-p^{2}}\right\| \leq \operatorname{const}(w) \lambda^{\frac{3}{2}}
$$

(norms of functions of $p$ as elements of $L^{2}$ ). Now we easily obtain in the $L^{2}\left(\mathbb{R}^{2}, \mathrm{~d} p \mathrm{~d} q\right)$-sense

$$
\begin{aligned}
& \mathrm{s}-\lim _{\lambda \rightarrow 0^{+}}\left\langle p\left|G_{\lambda}\left(w^{2}\right)-G_{0}\left(w^{2}\right)\right| q\right\rangle=-\frac{i w^{\sigma}}{\pi\left(1-\mathrm{e}^{2 i a w}\right)} \frac{(p q)^{\frac{1-\sigma}{2}}}{\left(w^{2}-p^{2}\right)\left(w^{2}-q^{2}\right)} \\
& \times\left[\mathrm{e}^{-i b q} \mathrm{e}^{i b p}-\mathrm{e}^{i a w} \mathrm{e}^{i b q} \mathrm{e}^{i b p}+\mathrm{e}^{i b q} \mathrm{e}^{-i b p}-\mathrm{e}^{i a w} \mathrm{e}^{-i b q} \mathrm{e}^{-i b p}\right] .
\end{aligned}
$$

We transform this to position representation and get

$$
\begin{aligned}
\mathrm{s}- & \lim _{\lambda \rightarrow 0^{+}}\left\langle z\left|G_{\lambda}\left(w^{2}\right)-G_{0}\left(w^{2}\right)\right| z^{\prime}\right\rangle \\
= & -\frac{\sigma}{2 i w\left(1-\mathrm{e}^{2 i a w}\right)}\left\{\left[\theta(b+z) \mathrm{e}^{i(b+z) w}+\sigma \theta(-b-z) \mathrm{e}^{-i(b+z) w}\right]\right. \\
& \times\left[\sigma \theta\left(-b-z^{\prime}\right) \mathrm{e}^{-i\left(b+z^{\prime}\right) w}+\theta\left(b+z^{\prime}\right) \mathrm{e}^{i\left(b+z^{\prime}\right) w}\right. \\
& \left.-\sigma \theta\left(b-z^{\prime}\right) \mathrm{e}^{i a w} \mathrm{e}^{i\left(b-z^{\prime}\right) w}-\theta\left(-b+z^{\prime}\right) \mathrm{e}^{i a w} \mathrm{e}^{-i\left(b-z^{\prime}\right) w}\right] \\
& +\left[\theta(-b+z) \mathrm{e}^{-i(b-z) w}+\sigma \theta(b-z) \mathrm{e}^{i(b-z) w}\right] \\
& \times\left[\sigma \theta\left(b-z^{\prime}\right) \mathrm{e}^{i\left(b-z^{\prime}\right) w}+\theta\left(-b+z^{\prime}\right) \mathrm{e}^{-i\left(b-z^{\prime}\right) w}\right. \\
& \left.\left.-\sigma \theta\left(-b-z^{\prime}\right) \mathrm{e}^{i a w} \mathrm{e}^{-i\left(b+z^{\prime}\right) w}-\theta\left(b+z^{\prime}\right) \mathrm{e}^{i a w} \mathrm{e}^{i\left(b+z^{\prime}\right) w}\right]\right\} .
\end{aligned}
$$

We shall use also the explicit form of the unperturbed Green function in this representation

$$
\left\langle z\left|G_{0}\left(w^{2}\right)\right| z^{\prime}\right\rangle=-\frac{i}{2 w}\left[\theta\left(z-z^{\prime}\right) \mathrm{e}^{i\left(z-z^{\prime}\right) w}+\theta\left(z^{\prime}-z\right) \mathrm{e}^{i\left(z^{\prime}-z\right) w}\right] .
$$

In this way we find

$$
\mathrm{s}-\lim _{\lambda \rightarrow 0^{+}}\left\langle z\left|G_{\lambda}\left(w^{2}\right)\right| z^{\prime}\right\rangle=\left\langle z\left|G^{B}\left(w^{2}\right)\right| z^{\prime}\right\rangle,
$$

where

$$
\begin{aligned}
\left\langle z\left|G^{B}\left(w^{2}\right)\right| z^{\prime}\right\rangle & \\
= & {\left[\left\langle z\left|G_{0}\left(w^{2}\right)\right| z^{\prime}\right\rangle+\sigma \frac{i}{2 w} \mathrm{e}^{-i\left(z+z^{\prime}+a\right) w}\right] \chi_{(-\infty,-b)}(z) \chi_{(-\infty,-b)}\left(z^{\prime}\right) } \\
& +\left[\left\langle z\left|G_{0}\left(w^{2}\right)\right| z^{\prime}\right\rangle+\sigma \frac{i}{2 w} \mathrm{e}^{i\left(z+z^{\prime}-a\right) w}\right] \chi_{(b,+\infty)}(z) \chi_{(b,+\infty)}\left(z^{\prime}\right) \\
& +\left[\left\langle z\left|G_{0}\left(w^{2}\right)\right| z^{\prime}\right\rangle+\frac{i}{w}\left(\frac{\cos (z w) \cos \left(z^{\prime} w\right)}{1+\sigma \mathrm{e}^{-i a w}}+\frac{\sin (z w) \sin \left(z^{\prime} w\right)}{1-\sigma \mathrm{e}^{-i a w}}\right)\right] \\
& \times \chi_{(-b, b)}(z) \chi_{(-b, b)}\left(z^{\prime}\right),
\end{aligned}
$$

and $\chi_{\Omega}$ is the characteristic function of the set $\Omega$. 
In the three regions $\left\langle z\left|G^{B}\left(w^{2}\right)\right| z^{\prime}\right\rangle$ differs from $\left\langle z\left|G_{0}\left(w^{2}\right)\right| z^{\prime}\right\rangle$ only by solutions of homogeneous equation and satisfies the boundary conditions

$$
\begin{aligned}
\left\langle \pm b\left|G^{B}\left(w^{2}\right)\right| z^{\prime}\right\rangle & =\left\langle z\left|G^{B}\left(w^{2}\right)\right| \pm b\right\rangle=0, \\
\left.\frac{\mathrm{d}}{\mathrm{d} z}\left\langle z\left|G^{B}\left(w^{2}\right)\right| z^{\prime}\right\rangle\right|_{z= \pm b} & =\left.\frac{\mathrm{d}}{\mathrm{d} z^{\prime}}\left\langle z\left|G^{B}\left(w^{2}\right)\right| z^{\prime}\right\rangle\right|_{z^{\prime}= \pm b}=0,
\end{aligned}
$$

so it is indeed the Green function of the Dirichlet/Neumann operator and therefore (25) is finally proven.

We now want to acquire some information on the rate at which the limit (25) is achieved. Using (30) one finds for any $\varphi, \eta \in L^{2}$

$$
\left(\varphi, G_{\lambda}\left(w^{2}\right) \eta\right)=\left(\varphi, G^{B}\left(w^{2}\right) \eta\right)+\left\{\begin{array}{l}
\left(1-I_{0}\right) O(\lambda)+O\left(\lambda^{3 / 2}\right) \\
O\left(\lambda^{1 / 2}\right)
\end{array}\right.
$$

The Neumann case turns out to be here, as in many other problems, more singular. However, we also note that if we assume that $\varphi$ and $\eta$ are in the domain of $h_{z}$ then the estimate (31) implies

$$
\left(\varphi, G_{\lambda}\left(w^{2}\right) \eta\right)=\left(\varphi, G^{B}\left(w^{2}\right) \eta\right)+I_{0} O(\lambda)+O\left(\lambda^{3 / 2}\right) .
$$

In the following two sections we treat unscaled models. The scaling is again considered in Sect. 6 .

\section{Spectral Analysis}

We add now some further assumptions on the choice of functions $f$. We denote for $k \in \mathbb{R}$

$$
I_{k}=\int \frac{M_{k}-M_{p}}{p^{2}-k^{2}} \mathrm{~d} p
$$

and demand that

$$
\begin{array}{ll}
0<I_{k} & \text { for } k \neq 0 \quad(\mathrm{D}, \mathrm{N}) \\
I_{k}<1 & \text { (D). }
\end{array}
$$

Note that by continuity $I_{0} \geq 0$ (this is the quantity introduced in (27)). We also denote

$$
\pi N_{k}=|k|^{\sigma}\left\{\sigma+\int \frac{q^{1-\sigma} M(q)-k^{1-\sigma} M(k)}{q^{2}-k^{2}} \mathrm{~d} q\right\}=\frac{1}{2}(1+\sigma)|k|^{\sigma}-|k| I_{k}
$$

The operators $h_{z a}^{2}$ are non-negative, and outside a compact set in $\mathbb{R}$ they act as $-\partial_{z}^{2}$. Therefore their continuous spectrum covers the whole positive axis and thus the spectrum is $\langle 0,+\infty)$. This does not resolve the question of point spectrum, and we treat it first.

The eigenvector equation

$$
h_{z a}^{2} \psi_{k}=k^{2} \psi_{k}, \quad \psi_{k} \in L^{2}(\mathbb{R}), \quad k \geq 0
$$


is solved in momentum space. It is easily seen that the distributional solution which is square-integrable at infinity must have the form

$$
\widehat{\psi}_{k}(p)=\frac{c_{b} \mathrm{e}^{-i b p}+c_{-b} \mathrm{e}^{i b p}}{p^{2}-k^{2}} p^{\frac{1-\sigma}{2}} \widehat{f}(p)
$$

with constants $c_{ \pm b}$ to be determined. Putting this form back into Eq. (40), one finds for $k>0$ that the constants $c_{ \pm b}$ have to satisfy the linear system

$$
N_{k} c_{+b}-M_{k} \sin (a k) c_{-b}=0, \quad M_{k} \sin (a k) c_{+b}-N_{k} c_{-b}=0,
$$

where the integration leading to coefficients $M_{k} \sin (a k)$ is carried out with the use of analyticity and asymptotic behavior of $M_{k}$ discussed in Appendix A. Now, for $k>0$ the conditions (37) and (38) imply $N_{k} \neq 0$, thus non-trivial solutions to the system (42) exist only if $M_{k} \sin (a k)= \pm N_{k}$, and in that cases (41) takes, respectively, the form

$$
\widehat{\psi}_{k}(p)=c_{-b} \frac{\mathrm{e}^{i b p} \mp \mathrm{e}^{-i b p}}{p^{2}-k^{2}} p^{\frac{1-\sigma}{2}} \widehat{f}(p) .
$$

The condition $\widehat{\psi}_{k} \in L^{2}(\mathbb{R})$ requires that $\widehat{f}( \pm k)=0$ or $\mathrm{e}^{i b k} \pm \mathrm{e}^{-i b k}=0$. Each of these cases implies $M_{k} \sin (a k)=0$, which shows that there are no eigenvectors for $k>0$.

For $k=0$ in Dirichlet case the solution (41) cannot be in $L^{2}$ as $\widehat{f}(0) \neq 0$. For Neumann case one finds that (41) is a distributional solution for any constants $c_{ \pm b}$, so we are free to choose them so as to satisfy the square-integrability of (41). This happens only for $c_{-b}=-c_{b}$ and then

$$
\widehat{\psi}_{0}(p)=\mathcal{N} \frac{\sin (b p)}{p} \widehat{f}(p),
$$

where $\mathcal{N}$ is a proportionality factor. The normalization condition $\left\|\widehat{\psi}_{0}\right\|_{L^{2}}=1$ gives

$$
|\mathcal{N}|^{2}=\frac{2}{a \pi M_{0}-I_{0}} .
$$

Summarizing, there are no bound states for Dirichlet case, however, for Neumann case there is one bound state, which corresponds to the zero eigenvalue, described by (43) and (44).

We now consider the continuous spectrum and for this purpose use the stationary scattering formalism. The improper eigenfunctions of scattering states in momentum representation are given in standard notation by

$$
\widehat{\psi}_{k}(p)=\langle p \mid k+\rangle=\delta(p-k)+\frac{\left\langle p\left|T\left(k^{2}+i 0\right)\right| k\right\rangle}{k^{2}-p^{2}+i 0},
$$

where $T\left(w^{2}\right)$ is the operator discussed in Sect. 2. The variable $k$ takes all values $k \neq 0$ and each spectrum point $k^{2}$ has two-fold degeneracy corresponding to $\pm k$. Taking into account the results of Sect. 2 , we can write

$$
\mathcal{T}\left(k^{2}+i 0\right)=\frac{|k|^{\sigma}}{i \pi}\left(\begin{array}{cc}
M_{k}-i N_{k} & M_{k} \mathrm{e}^{i a|k|} \\
M_{k} \mathrm{e}^{i a|k|} & M_{k}-i N_{k}
\end{array}\right)^{-1} .
$$


For later use we write this in two alternative forms. We introduce matrix notation

$$
\begin{aligned}
\mathcal{M}(k) & =\mathcal{F}_{k}^{\dagger} \mathcal{F}_{k}, \quad \mathcal{N}(k)=\frac{2|k|}{\pi}\left[\sigma \mathbb{1}+\mathcal{P} \int \frac{\mathcal{M}(p)}{p^{2}-k^{2}} \mathrm{~d} p\right] \\
\mathcal{L}(k) & =\mathcal{M}(k)+\mathcal{M}(-k)=2 k^{1-\sigma} M_{k}\left(\begin{array}{cc}
1 & \cos (a k) \\
\cos (a k) & 1
\end{array}\right) .
\end{aligned}
$$

and then

$$
\begin{aligned}
\mathcal{T}\left(k^{2}+i 0\right) & =\frac{2|k|}{\pi}(\mathcal{N}(k)+i \mathcal{L}(k))^{-1}, \\
\left|\left\langle p\left|T\left(k^{2}+i 0\right)\right| k\right\rangle\right|^{2} & =\operatorname{Tr}\left[\mathcal{M}(p) \mathcal{T}\left(k^{2}+i 0\right) \mathcal{M}(k) \mathcal{T}\left(k^{2}+i 0\right)^{\dagger}\right] .
\end{aligned}
$$

Finally, we calculate the inverse in (46) and write the result in the form

$$
\mathcal{T}\left(k^{2}+i 0\right)=\frac{1}{i \pi} \frac{|k|^{\sigma} s_{k}}{1-\left(q_{k} \mathrm{e}^{i a|k|}\right)^{2}}\left(\begin{array}{cc}
1 & -q_{k} \mathrm{e}^{i a|k|} \\
-q_{k} \mathrm{e}^{i a|k|} & 1
\end{array}\right),
$$

where

$$
s_{k}=\frac{1}{M_{k}-i N_{k}}, \quad q_{k}=\frac{M_{k}}{M_{k}-i N_{k}}=M_{k} s_{k} .
$$

Some properties of $s_{k}$ function are shown in Appendix A.

\section{Hilbert-Schmidt Properties}

In this section we show that our model satisfies the admissibility conditions (5) and (6). If we write $\mathrm{TR}_{\tau}$ for the l.h.s. of these two conditions, with $\tau=0$ for (5) and $\tau=1$ for (6), then

$$
\mathrm{TR}_{\tau}=\int_{\mathbb{R}^{2}}|p|^{\tau}\left|\left\langle p\left|h_{z a}-h_{z}\right| k+\right\rangle\right|^{2} \mathrm{~d} k \mathrm{~d} p+\frac{1-\sigma}{2} \int_{\mathbb{R}}|p|^{2+\tau}\left|\widehat{\psi}_{0}(p)\right|^{2} \mathrm{~d} p,
$$

where the first term results from the continuous spectrum space of $h_{z a}$ and the second is the bound state contribution in Neumann case. The second term is evidently finite (by (43)), and we restrict attention to the first one, which we denote $\mathrm{TR}_{\tau}^{\text {cont }}$. In momentum representation we have

$$
\left\langle p\left|h_{z a}-h_{z}\right| k+\right\rangle=(|k|-|p|) \widehat{\psi}_{k}(p)=\frac{\left\langle p\left|T\left(k^{2}+i 0\right)\right| k\right\rangle}{|p|+|k|},
$$

thus by change of variables for negative arguments we get

$$
\mathrm{TR}_{\tau}^{\text {cont }}=\int_{\mathbb{R}_{+}^{2}} \frac{p^{\tau}}{(p+k)^{2}} \sum_{ \pm \pm}\left|\left\langle \pm p\left|T\left(k^{2}+i 0\right)\right| \pm k\right\rangle\right|^{2} \mathrm{~d} k \mathrm{~d} p
$$

where the signs in 'bra' and 'ket' are uncorrelated and the sum is over all four possibilities. From now on we assume that $k, p \geq 0$. Using Eqs. (48), (50) we can write

$$
\sum_{ \pm \pm}\left|\left\langle \pm p\left|T\left(k^{2}+i 0\right)\right| \pm k\right\rangle\right|^{2}=\operatorname{Tr}\left[\mathcal{L}(p) \mathcal{T}\left(k^{2}+i 0\right) \mathcal{L}(k) \mathcal{T}\left(k^{2}+i 0\right)^{\dagger}\right]
$$


and substituting here (from (49))

$$
\mathcal{L}(k)=\frac{i k}{\pi}\left(\mathcal{T}\left(k^{2}+i 0\right)^{\dagger-1}-\mathcal{T}\left(k^{2}+i 0\right)^{-1}\right)
$$

we give Eq. (53) the form

$$
\mathrm{TR}_{\tau}^{\text {cont }}=\frac{2}{\pi} \int_{\mathbb{R}_{+}^{2}} \frac{k p^{\tau}}{(p+k)^{2}} \mathfrak{R e}\left[i \operatorname{Tr}\left[\mathcal{L}(p) \mathcal{T}\left(k^{2}+i 0\right)\right]\right] \mathrm{d} k \mathrm{~d} p .
$$

With the use of (48) and (51) we have

$$
k \mathfrak{R e}\left[i \operatorname{Tr}\left[\mathcal{L}(p) \mathcal{T}\left(k^{2}+i 0\right)\right]\right]=\frac{4}{\pi} p^{1-\sigma} k^{1+\sigma} M_{p} \mathfrak{R e}\left[s_{k} \frac{1-\cos (a p) q_{k} \mathrm{e}^{i a k}}{1-\left(q_{k} \mathrm{e}^{i a k}\right)^{2}}\right]
$$

Writing out the real part gives us the appropriate behavior of the nominator for $k=0$ and the whole expression becomes proportional to $M_{k}$. Using the estimates (92), (91) and (87), one finds that $\mathrm{TR}_{\tau}^{\text {cont }}$ are finite and the admissibility conditions (5) and (6) are satisfied.

\section{Scaling}

We return to the scaling transformation to view it from a different point. If we make the dependence of the potential $V$ on $a$ explicit by writing it as $V_{a}$ and the rescaled potential as $V_{a, \lambda}$ then we have

$$
V_{a, \lambda}\left(z, z^{\prime}\right)=\lambda^{-3} V_{a / \lambda}\left(z / \lambda, z^{\prime} / \lambda\right) .
$$

It is then an easy exercise to show that this implies a simple scaling law of the eigenfunctions (43) and (45):

$$
\widehat{\psi}_{k, b}^{\lambda}(p)=\lambda \widehat{\psi}_{\lambda k, b / \lambda}(\lambda p), \quad \widehat{\psi}_{0, b}^{\lambda}(p)=\lambda^{1 / 2} \widehat{\psi}_{0, b / \lambda}(\lambda p)
$$

(different powers of $\lambda$ reflect different normalizations: to the Dirac delta and to unity, respectively). Denoting the scaled versions of (52), with explicit dependence on $a$, by $\mathrm{TR}_{\tau, a}^{\lambda}$ we find

$$
\mathrm{TR}_{\tau, a}^{\lambda}=\lambda^{-2-\tau} \mathrm{TR}_{\tau, a / \lambda}
$$

Thus the admissibility conditions are satisfied also for the rescaled potential (but not in the limit). In the same way one obtains the scaling law for the Casimir energy:

$$
\varepsilon_{a}^{\lambda}=\lambda^{-3} \varepsilon_{a / \lambda}
$$

Therefore to identify the scaling behavior of the energy in the limit it is sufficient to expand the unscaled energy in inverse powers of $a$ up to the third order. This will be done in the next section. 


\section{The Energy}

In this section we prove the following expansion of Casimir energy for large $a$ :

$$
\begin{aligned}
& \varepsilon_{a}=\varepsilon_{\infty}-\frac{\pi^{2}}{1440 a^{3}}+o\left(a^{-3}\right), \quad(\mathrm{D}) \\
& \varepsilon_{a}=\varepsilon_{\infty}+\frac{1}{48 \pi M_{0} a^{2}}+\frac{I_{0}}{8 \pi^{2} M_{0}^{2} a^{3}}\left(\frac{\zeta(3)}{\pi^{2}}+\frac{1}{3}\right)-\frac{\pi^{2}}{1440 a^{3}}+o\left(a^{-3}\right) .
\end{aligned}
$$

We postpone the discussion of this result to the concluding section and here only note that there are functions in our class for which $I_{0}=0$, and then the $a^{-3}$-term has the known universal form.

The two conditions (5) and (6), considered in Sect. 5, imply already finiteness of the Casimir energy per unit area (7), as mentioned in the Introduction. This can be easily seen: we observe that conditions (5) and (6) mean that $\Delta=h_{z a}-h_{z}$ and $h_{z}^{1 / 2} \Delta$ are Hilbert-Schmidt operators. Also, from

$$
\Delta h_{z a} \Delta=\Delta h_{z} \Delta+\Delta^{3}
$$

we infer that $h_{z a}^{1 / 2} \Delta$ is HS as well, which is sufficient for the claim.

The expression (7) closely parallels that of the condition (6) and can be written in analogy to (52). We split the trace in (7) into two terms and calculate these traces in $h_{z a}$ or $h_{z}$ (improper) basis in the first and second line below, respectively. Then we insert the spectral decomposition of the operators $h_{z}$ or $h_{z a}$, respectively. In this way we get

$$
\begin{aligned}
& \operatorname{Tr}\left[2 \Delta h_{z} \Delta\right]=2 \int_{\mathbb{R}^{2}}|p|\left|\left\langle p\left|h_{z a}-h_{z}\right| k+\right\rangle\right|^{2} \mathrm{~d} k \mathrm{~d} p+(1-\sigma) \int_{\mathbb{R}}|p|^{3}\left|\widehat{\psi}_{0}(p)\right|^{2} \mathrm{~d} p, \\
& \operatorname{Tr}\left[\Delta h_{z a} \Delta\right]=\int_{\mathbb{R}^{2}}|k|\left|\left\langle p\left|h_{z a}-h_{z}\right| k+\right\rangle\right|^{2} \mathrm{~d} k \mathrm{~d} p .
\end{aligned}
$$

Therefore the expression (7) may be written in the form

$$
\varepsilon_{a}=\frac{1}{24 \pi} \int_{\mathbb{R}^{2}}(2|p|+|k|)\left|\left\langle p\left|h_{z a}-h_{z}\right| k+\right\rangle\right|^{2} \mathrm{~d} k \mathrm{~d} p+\frac{1-\sigma}{24 \pi} \int_{\mathbb{R}}|p|^{3}\left|\widehat{\psi}_{0}(p)\right|^{2} \mathrm{~d} p .
$$

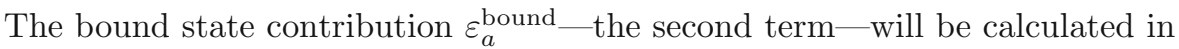
the Neumann case subsection. Following the same steps as in Sect. 5 and using (55) we give the continuous spectrum contribution the form

$$
\varepsilon_{a}^{\text {cont }}=\frac{1}{3 \pi^{3}} \int_{\mathbb{R}_{+}^{2}} \chi(k, p) M_{p} \mathfrak{R e}\left[s_{k} \frac{1-\cos (a p) q_{k} \mathrm{e}^{i a k}}{1-\left(q_{k} \mathrm{e}^{i a k}\right)^{2}}\right] \mathrm{d} k \mathrm{~d} p,
$$

where

$$
\chi(k, p)=\frac{k^{1+\sigma} p^{1-\sigma}(2 p+k)}{(p+k)^{2}} .
$$


We write this as the limit for $\epsilon \rightarrow 0^{+}$of the integral restricted to $k \in\langle\epsilon,+\infty)$ and expand the denominator into geometric power series (note that $\left|q_{k}\right|<1$ for $k>0$ )

$$
\frac{1}{1-\left(q_{k} \mathrm{e}^{i a k}\right)^{2}}=\sum_{n=0}^{\infty}\left(q_{k} \mathrm{e}^{i a k}\right)^{2 n},
$$

getting

$$
\begin{aligned}
\varepsilon_{a}^{\mathrm{cont}}= & \frac{1}{3 \pi^{3}} \lim _{\epsilon \rightarrow 0^{+}} \int_{0}^{\infty} M_{p} \int_{\epsilon}^{\infty} \chi(k, p) \mathfrak{R e}\left[s_{k} \sum_{n \in 2 \mathbb{N}_{0}} q_{k}^{n} \mathrm{e}^{i n a k}\right. \\
& \left.-s_{k} \cos (a p) \sum_{n \in 2 \mathbb{N}-1} q_{k}^{n} \mathrm{e}^{i n a k}\right] \mathrm{d} k \mathrm{~d} p .
\end{aligned}
$$

We write the $n=0$ term separately as

$$
\varepsilon_{\infty}=\frac{1}{3 \pi^{3}} \int_{\mathbb{R}_{+}^{2}} \chi(k, p) M_{p} M_{k}\left|s_{k}\right|^{2} \mathrm{~d} k \mathrm{~d} p .
$$

For other terms we observe that

$$
\sum_{n=1}^{N}\left|q_{k} \mathrm{e}^{i a k}\right|^{2 n} \leq \frac{\left|s_{k}\right|^{2} M_{k}^{2}}{1-\left|q_{k}\right|^{2}} \leq \text { const } M_{k}^{2} \frac{1+k^{m-2 \sigma}}{k^{m}} \quad\left\{\begin{array}{l}
m=2 \\
m \geq 2
\end{array}\right.
$$

[see (93) and (91)], so we can pull the infinite sum sign outside the integral by the Lebesgue dominated convergence theorem to obtain

$$
\begin{aligned}
\varepsilon_{a}^{\text {cont }}= & \varepsilon_{\infty}+\frac{1}{6 \pi^{3}} \lim _{\epsilon \rightarrow 0^{+}} \sum_{n \in 2 \mathbb{N}} \int_{0}^{\infty} M_{p}\left[\int_{\epsilon}^{\infty} \chi(k, p) s_{k}\left(q_{k} \mathrm{e}^{i a k}\right)^{n} \mathrm{~d} k+\text { c.c. }\right] \mathrm{d} p \\
& -\frac{1}{6 \pi^{3}} \lim _{\epsilon \rightarrow 0^{+}} \sum_{n \in 2 \mathbb{N}-1} \int_{0}^{\infty} M_{p} \cos (a p)\left[\int_{\epsilon}^{\infty} \chi(k, p) s_{k}\left(q_{k} \mathrm{e}^{i a k}\right)^{n} \mathrm{~d} k+\text { c.c. }\right] \mathrm{d} p .
\end{aligned}
$$

We now split the analysis into separate cases.

\subsection{Dirichlet Case $(\sigma=1)$}

In this case when $(62)$ is multiplied by $\chi(k, p)$ the $k^{-2}$ singularity in $k=0$ is cancelled by $k^{2}$ from the function $\chi$. Therefore here the intermediate step with nonzero $\epsilon$ is not needed and (63) should be read with $\epsilon=0$. Moreover, there is no bound state here, so this formula represents the total Casimir energy $\varepsilon_{a}$. If we write $(n a)^{3} \mathrm{e}^{i n a k}=\left(-i \partial_{k}\right)^{3} \mathrm{e}^{i n a k}$ and integrate three times by parts we find

$$
(n a)^{3} \int_{0}^{\infty} \chi(k, p) s_{k}\left(q_{k} \mathrm{e}^{i a k}\right)^{n} \mathrm{~d} k=-i \frac{4 M_{p}}{M_{0} p}-i \int_{0}^{\infty} \partial_{k}^{3}\left(\chi(k, p) s_{k} q_{k}^{n}\right) \mathrm{e}^{i n a k} \mathrm{~d} k
$$


and then obtain (the first term on the r.h.s. above is imaginary and falls out)

$$
\begin{aligned}
\varepsilon_{a}-\varepsilon_{\infty}= & \frac{i}{6 \pi^{3} a^{3}}\left\{-\sum_{n \in 2 \mathbb{N}} \frac{1}{n^{3}} \int_{\mathbb{R}_{+}^{2}} M_{p} \partial_{k}^{3}\left(\chi(k, p) s_{k} q_{k}^{n}\right) \mathrm{e}^{i n a k} \mathrm{~d} k \mathrm{~d} p\right. \\
& \left.+\sum_{n \in 2 \mathbb{N}-1} \frac{1}{n^{3}} \int_{\mathbb{R}_{+}^{2}} M_{p} \cos (a p) \partial_{k}^{3}\left(\chi(k, p) s_{k} q_{k}^{n}\right) \mathrm{e}^{i n a k} \mathrm{~d} k \mathrm{~d} p\right\}+ \text { c.c.. }
\end{aligned}
$$

Let now $\Omega$ be the intersection of $\mathbb{R}_{+}^{2}$ with an arbitrary neighborhood of zero. We now use the results of Appendix B to infer that the following successive three operations on this formula lead only to the neglect of terms of order $o\left(a^{-3}\right)$ :

(i) replacement of $\partial_{k}^{3}\left(\chi(k, p) s_{k} q_{k}^{n}\right)$ by $s_{k} q_{k}^{n} \partial_{k}^{3} \chi(k, p)$;

(ii) restriction of the integration region to $\Omega$;

(iii) replacement (in the restricted region) of $M_{p} s_{k} q_{k}^{n}$ by $M_{0} s_{0} q_{0}^{n}=1$.

In this way we arrive at

$$
\begin{aligned}
\varepsilon_{a}= & \varepsilon_{\infty}+\frac{2}{\pi^{3} a^{3}} \sum_{n \in 2 \mathbb{N}} \frac{1}{n^{3}} \int_{\Omega} \frac{p^{2}(k-3 p)}{(k+p)^{5}} \sin (n a k) \mathrm{d} k \mathrm{~d} p \\
& -\frac{2}{\pi^{3} a^{3}} \sum_{n \in 2 \mathbb{N}-1} \frac{1}{n^{3}} \int_{\Omega} \frac{p^{2}(k-3 p)}{(k+p)^{5}} \cos (a p) \sin (n a k) \mathrm{d} k \mathrm{~d} p+o\left(\frac{1}{a^{3}}\right) .
\end{aligned}
$$

The integrals are bounded uniformly with respect to $a$. It is now sufficient to show that they have well defined limits for $a \rightarrow \infty$; then those limits determine the $a^{-3}$-term.

Consider the integrals (with $k, p>0$ )

$$
C(n, \ell, a)=\int_{k+p \leq 1}\left(\frac{p^{2}}{(k+p)^{4}}-\frac{4 p^{3}}{(k+p)^{5}}\right) \sin (n a k+\ell a p) \mathrm{d} p \mathrm{~d} k, \quad \ell=0, \pm 1 .
$$

It is easy to show that with the choice $\Omega=\{k, p>0, k+p \leq 1\}$ the integral in the first line of (65) is this integral with $\ell=0$, while the integral in the second line of $(65)$ is one half of the sum of integrals with $\ell= \pm 1$. By the change of variables $r=a(k+p), t=p /(k+p)$ we bring this to the form

$$
C(n, \ell, a)=\int_{0}^{1}\left(t^{2}-4 t^{3}\right) \int_{0}^{a} \frac{1}{r} \sin [r(n+t(\ell-n))] \mathrm{d} r \mathrm{~d} t,
$$

and find

$$
\lim _{a \rightarrow \infty} C(n, \ell, a)= \begin{cases}-\frac{\pi}{3} & \text { for } \ell=0,1 \\ \frac{\pi}{3}\left(1+\frac{n^{3}}{(n+1)^{3}}-\frac{3 n^{4}}{(n+1)^{4}}\right) & \text { for } \ell=-1\end{cases}
$$


Using these results we get finally

$$
\begin{aligned}
\varepsilon_{a} & =\varepsilon_{\infty}-\frac{1}{3 \pi^{2} a^{3}}\left[\sum_{n \in 2 \mathbb{N}} \frac{2}{n^{3}}+\sum_{n \in 2 \mathbb{N}-1}\left(\frac{1}{(n+1)^{3}}-\frac{3 n}{(n+1)^{4}}\right)\right]+o\left(\frac{1}{a^{3}}\right) \\
& =\varepsilon_{\infty}-\frac{\zeta(4)}{16 \pi^{2} a^{3}}+o\left(\frac{1}{a^{3}}\right)=\varepsilon_{\infty}-\frac{\pi^{2}}{1440 a^{3}}+o\left(\frac{1}{a^{3}}\right),
\end{aligned}
$$

where $\varepsilon_{\infty}$ is defined in (61).

\subsection{Neumann Case $(\sigma=-1)$}

We now use formula (63) with the replacement in notation $q_{k} \mathrm{e}^{i a k}=\tilde{q}_{k} \mathrm{e}^{i \tilde{a} k}$, where

$$
\alpha=\frac{I_{0}}{\pi M_{0}}, \quad \tilde{a}=a-\alpha, \quad \tilde{q}_{k}=q_{k} \mathrm{e}^{i \alpha k}
$$

which has the technical advantage that $\tilde{q}_{0}^{\prime}=0$. After this modification the general scheme is very similar to the Dirichlet case. Integration by parts gives expansion in $1 / \tilde{a}$ but at the end we shall translate it to the $1 / a$ expansion. Integrating by parts we obtain boundary terms, for which in the present case the addition of c.c. terms must be taken into account before the limit $\epsilon \rightarrow 0^{+}$ is performed. For example, the first integration by parts in $k$ in the first line in (63) gives a term proportional to (before $p$-integration)

$$
\begin{aligned}
& \lim _{\epsilon \rightarrow 0^{+}} \sum_{n \in 2 \mathbb{N}} \frac{1}{n} \chi(\epsilon, p) \mathfrak{I m}\left[s_{k} \tilde{q}_{k}^{n}(\epsilon) \mathrm{e}^{i n \tilde{a} \epsilon}\right] \\
& \quad=-\lim _{\epsilon \rightarrow 0^{+}} \chi(\epsilon, p) \mathfrak{I m}\left[s_{\epsilon} \ln \left(1-\tilde{q}_{\epsilon}^{2} \mathrm{e}^{2 i \tilde{a} \epsilon}\right)\right]=\frac{\pi p}{M_{0}},
\end{aligned}
$$

where in the last equality we used the fact that in the neighborhood of zero $|\ln | 1-\tilde{q}_{\epsilon}^{2} \mathrm{e}^{2 i \tilde{a} \epsilon}|| \leq \ln \frac{\text { const }(a)}{\epsilon}$ [see (92)]. After integrating by parts three times in similar way we get the expression

$$
\begin{aligned}
\lim _{\epsilon \rightarrow 0^{+}} & {\left[\sum_{n \in 2 \mathbb{N}} \int_{\epsilon}^{\infty} \chi(k, p) s_{k} \tilde{q}_{k}^{n} \mathrm{e}^{i n \tilde{a} k} \mathrm{~d} k+\text { c.c. }\right]=-\frac{\pi p}{\tilde{a} M_{0}}+\frac{\pi^{2}}{4 \tilde{a}^{2} M_{0}}+\frac{3 I_{0} \zeta(3)}{2 \tilde{a}^{3} \pi M_{0}^{2}} } \\
& -\lim _{\epsilon \rightarrow 0^{+}}\left[\sum_{n \in 2 \mathbb{N}} \frac{1}{(i n \tilde{a})^{3}} \int_{\epsilon}^{\infty} \partial_{k}^{3}\left(\chi(k, p) s_{k} \tilde{q}_{k}^{n}\right) \mathrm{e}^{i n \tilde{a} k} \mathrm{~d} k+\text { c.c. }\right] .
\end{aligned}
$$

For the sum over odd natural numbers the computations are similar. The integrals over $p$ we treat in a similar way as in the Dirichlet case (steps (i) to (iii), for their permissibility here see Appendix B). The integrals of $\partial_{k}^{3} \chi$ over the neighborhood of zero go similarly as in the Dirichlet case. In this way we find

$$
\varepsilon_{a}^{\mathrm{cont}}=\varepsilon_{\infty}-\frac{C_{M}}{6 \pi^{2} M_{0} \tilde{a}}+\frac{1}{48 \pi M_{0} \tilde{a}^{2}}-\frac{1}{\tilde{a}^{3}}\left[\frac{1}{6 \pi^{2}}-\frac{I_{0} \zeta(3)}{8 \pi^{4} M_{0}^{2}}+\frac{\pi^{2}}{1440}\right]+o\left(\frac{1}{\tilde{a}^{3}}\right),
$$


where

$$
C_{M}=\int_{0}^{\infty} p M_{p} \mathrm{~d} p .
$$

In the calculation we used the relations

$$
\int_{0}^{\infty} M_{p} \mathrm{~d} p=\frac{1}{2}, \quad \int_{0}^{\infty} p M_{p} \cos (a p) \mathrm{d} p=-\frac{M_{0}}{\tilde{a}^{2}}+O\left(\frac{1}{\tilde{a}^{3}}\right) .
$$

The first equality follows from normalization of $f$. We now return to the bound state contribution to the energy. From the second term of (59), (43) and (44), using (66) we get

$$
\varepsilon_{a}^{\text {bound }}=\frac{C_{M}}{6 \pi^{2} M_{0} \tilde{a}}+\frac{1}{6 \pi^{2} \tilde{a}^{3}}+o\left(\frac{1}{\tilde{a}^{3}}\right) .
$$

Adding the two contributions and changing the expansion parameter to $a$ we obtain (58).

\section{Local Properties}

In this section we consider the local algebras of fields supported outside the regions of support of the potential $V$. For the initial (unscaled) models this means that the $z$-support of fields is outside the set $\langle-b-R,-b+R\rangle \cup$ $\langle b-R, b+R\rangle$, but for $\lambda \rightarrow 0$ eventually every support outside the planes $z= \pm b$ is admitted. Fields thus supported are also in the algebra of fields of the models with sharp boundary conditions at $z= \pm b$, so one can also consider sharp boundary conditions for them.

We recall from [1] that we use the initial value fields (smeared on a Cauchy surface of constant time) $\Phi(V)$, where $V$ is a pair of real test functions $(v, u)$, and $X(u)=\Phi(0, u), P(v)=\Phi(v, 0)$ have the interpretation of canonical variables. For the present choice of the algebras the test functions are assumed to be in the space of smooth functions of compact support outside $z= \pm b$, which we denote by $\mathcal{D}_{b}$. The algebra of fields is formulated, more precisely, in Weyl form, which means that rather than $\Phi(V)$ elements $W(V)=\exp [i \Phi(V)]$ are used, and the fields $\Phi$ are defined on the level of specific representations. The states on the algebra are given as normalized positive linear functionals on the algebra of Weyl elements.

With the Hamiltonians of the perpendicular motion $h_{z a}$ and $h_{z a}^{B}$ as defined in (13) and (24) we denote

$$
h_{a}^{2}=h_{z a}^{2}+h_{\perp}^{2}, \quad\left[h_{a}^{B}\right]^{2}=\left[h_{z a}^{B}\right]^{2}+h_{\perp}^{2},
$$

where $-h_{\perp}^{2}$ is the two-dimensional Laplacian in the directions parallel to the plates. Then the ground states of the fields corresponding to the models proposed in the present article and to the sharp boundary conditions are given, respectively, by

$$
\omega_{a}(W(V))=\exp \left[-\frac{1}{4}\left\|j_{a}(V)\right\|^{2}\right], \quad \omega_{a}^{B}(W(V))=\exp \left[-\frac{1}{4}\left\|j_{a}^{B}(V)\right\|^{2}\right],
$$


where

$$
j_{a}(V)=h_{a}^{1 / 2} v-i h_{a}^{-1 / 2} u, \quad j_{a}^{B}(V)=\left[h_{a}^{B}\right]^{1 / 2} v-i\left[h_{a}^{B}\right]^{-1 / 2} u .
$$

(To be precise, to obtain formula (7) for the Casimir energy we start in [2] with the directions parallel to the plates restricted to a box, whose size then tends to infinity. This may be shown to reproduce the states given above, but we omit this step here.) The ground states $\omega_{a, \lambda}$ of the scaled models are defined analogously with the use of $h_{a, \lambda}$.

We show in this section that the scaled states reproduce in the weak limit the sharp boundary state:

$$
\lim _{\lambda \rightarrow 0} \omega_{a, \lambda}(W(V))=\omega_{a}^{B}(W(V)) .
$$

Also, we consider the limit of the local energy density.

\subsection{Local Limit of States}

There is $\left\|j_{a}(V)\right\|^{2}=\left(v, h_{a} v\right)+\left(u, h_{a}^{-1} u\right)$, thus to prove (70) it is sufficient to show that $\left(\varphi, h_{a, \lambda}^{ \pm 1} \psi\right)$ tend to $\left(\varphi,\left[h_{a}^{B}\right]^{ \pm 1} \psi\right)$ for $\lambda \rightarrow 0$ and $\varphi, \psi \in \mathcal{D}_{b}$. For such $\psi$ one has

$$
h_{a, \lambda}^{2} \psi=\left[h_{a}^{B}\right]^{2} \psi=h^{2} \psi \equiv-\Delta \psi
$$

for sufficiently small $\lambda$. Therefore $\left(\varphi, h_{a, \lambda} \psi\right)=-\left(\varphi, h_{a, \lambda}^{-1} \Delta \psi\right)$ and similarly for $h_{a}^{B}$, and the problem is reduced to the $h_{a, \lambda}^{-1}$-case. Moreover, $\varphi$ and $\psi$ are in the domain of $h_{\perp}^{-1 / 2}$, so we can write

$$
\left(\varphi, h_{a, \lambda}^{-1} \psi\right)=\left(\left(h_{\perp}^{-1 / 2} \varphi\right), h_{\perp}^{1 / 2} h_{a, \lambda}^{-1} h_{\perp}^{1 / 2}\left(h_{\perp}^{-1 / 2} \psi\right)\right)
$$

and similarly for $h_{a}^{B}$. In this way the problem is reduced to the following:

$$
\mathrm{w}-\lim _{\lambda \rightarrow 0} h_{\perp}^{1 / 2} h_{a, \lambda}^{-1} h_{\perp}^{1 / 2}=h_{\perp}^{1 / 2}\left[h_{a}^{B}\right]^{-1} h_{\perp}^{1 / 2} .
$$

To show this, we first observe that $\left\|h_{\perp}^{1 / 2} h_{a, \lambda}^{-1} h_{\perp}^{1 / 2}\right\| \leq 1$, so it is sufficient to perform the limit between vectors from the total set of the form $\chi(\vec{x})=\chi_{\perp}\left(\vec{x}_{\perp}\right) \chi_{z}\left(x^{3}\right)$. Using the spectral representation of $h_{\perp}^{2}$ one has

$$
\left(\chi, h_{\perp}^{1 / 2} h_{a, \lambda}^{-1} h_{\perp}^{1 / 2} \rho\right)=\int\left(\chi_{z}, \frac{\left|\vec{p}_{\perp}\right|}{\sqrt{h_{z a, \lambda}^{2}+\vec{p}_{\perp}^{2}}} \rho_{z}\right) \overline{\widehat{\chi_{\perp}}\left(\vec{p}_{\perp}\right)} \widehat{\rho} \perp_{\perp}\left(\vec{p}_{\perp}\right) \mathrm{d}^{2} p_{\perp}
$$

The scalar product under the integral is bounded by $\left\|\chi_{z}\right\|\left\|\rho_{z}\right\|$ and for each $\vec{p}_{\perp}$, by the result of Sect. 3, tends to analogous expression with $h_{z a, \lambda}$ replaced by $h_{z a}^{B}$. This is sufficient to perform the limit, which ends the proof. 


\subsection{Local Energy Density}

The state $\omega_{a}$ (unlike the state $\omega_{a}^{B}$ ) is defined on the whole algebra. In the language used in [1] the energy density in this state (properly normally ordered with respect to the vacuum) is given in the whole space by point-splitting procedure by

$$
\mathcal{E}_{a}(\vec{x})=T_{a}(\vec{x}, \vec{x}),
$$

where $T_{a}(\vec{x}, \vec{y})$ is the distribution defined by

$$
T_{a}(\varphi, \psi)=\frac{1}{4}\left(\varphi,\left(h_{a}-h\right) \psi\right)+\frac{1}{4}\left(\vec{\nabla} \varphi,\left(h_{a}^{-1}-h^{-1}\right) \vec{\nabla} \psi\right),
$$

with scalar product between the two gradients understood in the second term. The test functions are taken to be real. If one takes into account the translational symmetry in the directions parallel to the plates one realizes that the $\vec{x}_{\perp}, \vec{y}_{\perp}$-dependence of $T_{a}$ may be only through the difference $\vec{x}_{\perp}-\vec{y}_{\perp}$. The removal of point-splitting in these directions means putting this variable equal to zero, or integrating the 2-dimensional Fourier transform of $T_{a}$ over all space of $\vec{p}_{\perp}$-variables of the spectral representation of $h_{\perp}$. In this way one finds (now $x, y$ are variables in the direction orthogonal to plates and $\varphi, \psi$ are one-dimensional)

$$
\mathcal{E}_{a}(x)=T_{z a}(x, x),
$$

where

$$
\begin{aligned}
T_{z a}(\varphi, \psi)= & \frac{1}{16 \pi^{2}} \int\left\{\left(\varphi,\left[\left(h_{z a}^{2}+\vec{p}_{\perp}^{2}\right)^{1 / 2}-\left(h_{z}^{2}+\vec{p}_{\perp}^{2}\right)^{1 / 2}\right] \psi\right)\right. \\
& +\vec{p}_{\perp}^{2}\left(\varphi,\left[\left(h_{z a}^{2}+\vec{p}_{\perp}^{2}\right)^{-1 / 2}-\left(h_{z}^{2}+\vec{p}_{\perp}^{2}\right)^{-1 / 2}\right] \psi\right) \\
& \left.+\left(\varphi^{\prime},\left[\left(h_{z a}^{2}+\vec{p}_{\perp}^{2}\right)^{-1 / 2}-\left(h_{z}^{2}+\vec{p}_{\perp}^{2}\right)^{-1 / 2}\right] \psi^{\prime}\right)\right\} \mathrm{d}^{2} p_{\perp},
\end{aligned}
$$

with prime in $\varphi^{\prime}, \psi^{\prime}$ denoting the derivative. The integral is easily carried out explicitly and one finds

$$
T_{z a}(\varphi, \psi)=\frac{1}{24 \pi}\left(\varphi,\left(h_{z a}^{3}-h_{z}^{3}\right) \psi\right)-\frac{1}{8 \pi}\left(\varphi^{\prime},\left(h_{z a}-h_{z}\right) \psi^{\prime}\right) .
$$

Our objective is to find the limit of the energy density in the scaled models. Thus following the introductory remarks of the present section we assume now the supports of $\varphi, \psi$ to be outside the set $\langle-b-R,-b+R\rangle \cup\langle b-R, b+R\rangle$. For such functions there is $h_{z a}^{2} \psi=h_{z}^{2} \psi$, so

$$
T_{z a}(\varphi, \psi)=-\frac{1}{24 \pi}\left(\varphi,\left(h_{z a}-h_{z}\right) \psi^{\prime \prime}\right)-\frac{1}{8 \pi}\left(\varphi^{\prime},\left(h_{z a}-h_{z}\right) \psi^{\prime}\right) .
$$

Consider the general element $\left(\varphi,\left(h_{z a}-h_{z}\right) \psi\right)$ with test functions in the assumed class. For any non-negative real numbers $a, b$ one has the identity

$$
a-b=-\frac{2}{\pi} \int_{0}^{\infty}\left\{\frac{1}{\left(a^{2}+r^{2}\right)}-\frac{1}{\left(b^{2}+r^{2}\right)}\right\} r^{2} \mathrm{~d} r .
$$


Using this and the spectral representations of $h_{z a}$ and $h_{z}$ one finds

$$
\left(\varphi,\left(h_{z a}-h_{z}\right) \psi\right)=\frac{2}{\pi} \int_{0}^{\infty}\left(\varphi,\left[G\left(-r^{2}\right)-G_{0}\left(-r^{2}\right)\right] \psi\right) r^{2} \mathrm{~d} r .
$$

To find the integral kernel $\left[G\left(-r^{2}\right)-G_{0}\left(-r^{2}\right)\right](x, y)$ of $\left[G\left(-r^{2}\right)-G_{0}\left(-r^{2}\right)\right]$, one needs only to transform the kernel (21) to the position space:

$$
\begin{aligned}
& {\left[G\left(-r^{2}\right)-G_{0}\left(-r^{2}\right)\right](x, y)} \\
& \quad=\frac{1}{2 \pi} \int \mathrm{e}^{i p x} \frac{\mathcal{F}_{p}}{\left(p^{2}+r^{2}\right)} \mathrm{d} p \mathcal{T}\left(-r^{2}\right) \int \mathrm{e}^{-i q y} \frac{\mathcal{F}_{q}^{\dagger}}{\left(q^{2}+r^{2}\right)} \mathrm{d} q .
\end{aligned}
$$

For $x, y$ in the assumed region the Fourier integrals may be closed to contour integrals in the complex plane (the half-circular contributions vanish either in the upper or the lower half-plane due to the bound (83)) and evaluated by residues. Because eventually we are interested in removal of point-splitting we assume that $x$ and $y$ are in the same connected part of the region considered. We denote $\mathcal{T}\left(-r^{2}\right)=\left(\begin{array}{ll}A & B \\ B & A\end{array}\right)$, where $A$ and $B$ are functions of $r$, and get

$$
\begin{aligned}
& {\left[G\left(-r^{2}\right)-G_{0}\left(-r^{2}\right)\right](x, y)=\frac{\pi}{r^{1+\sigma}}|\widehat{f}(i r)|^{2}} \\
& \quad \times\left\{\begin{array}{cc}
\mathrm{e}^{-|x+y| r}[A \cosh (a r)+B] & \text { for } x, y>b+R \text { or } x, y<-b-R, \\
\mathrm{e}^{-a r}[A \cosh ((x+y) r)+\sigma B \cosh ((x-y) r)] & \text { for } x, y \in(-b+R, b-R) .
\end{array}\right.
\end{aligned}
$$

We use this integral kernel in (77), evaluate derivatives by parts and remove the point-splitting. In this way we find in the assumed regions

$$
\begin{aligned}
& \mathcal{E}_{a}(x)=T_{z a}(x, x) \\
& =-\frac{1}{6 \pi} \int_{0}^{\infty}\left\{\begin{array}{cl}
2 \mathrm{e}^{-2|x| r}(A \cosh (a r)+B) \\
\mathrm{e}^{-a r}(2 A \cosh (2 x r)-\sigma B)
\end{array}\right\}|\widehat{f}(i r)|^{2} r^{3-\sigma} \mathrm{d} r, \quad \begin{array}{l}
|x|>b+R, \\
|x|<b-R .
\end{array}
\end{aligned}
$$

We now want to consider the limit of this local energy in the scaled version of the model. Because of the appropriate convergence of the integral this limit may be performed inside the integral. The scaling of $\widehat{f}(i r)$ and $\mathcal{T}\left(-r^{2}\right)$ follows from Sect. 3. As for the scaled model the excluded position set shrinks to ||$x|-b| \leq \lambda R$, in the limit all $x \neq \pm b$ are admitted. A straightforward calculation yields

$$
\begin{aligned}
& \lim _{\lambda \rightarrow 0^{+}} \mathcal{E}_{a, \lambda}(x) \\
& \quad=-\frac{\sigma}{6 \pi^{2}} \int_{0}^{\infty}\left\{\begin{array}{cl}
\mathrm{e}^{-(2|x|-a) r} & \text { for }|x|>b, \\
\mathrm{e}^{(2 x-a) r}+\mathrm{e}^{-(2 x+a) r}+\sigma \mathrm{e}^{-2 a r} & \text { for }|x|<b .
\end{array}\right\} r^{3} \mathrm{~d} r,
\end{aligned}
$$


With the help of Eq. (94) in Appendix A we get

$$
\begin{aligned}
\mathcal{E}_{a}^{B}(x) \equiv & \lim _{\lambda \rightarrow 0^{+}} \mathcal{E}_{a, \lambda}(x) \\
& = \begin{cases}-\frac{\sigma}{16 \pi^{2}(|x|-b)^{4}} & \text { for }|x|>b, \\
-\frac{\pi^{2}}{1440 a^{4}}-\sum_{n \in(2 \mathbb{Z}+1)} \frac{\sigma}{16 \pi^{2}(n b-x)^{4}} & \text { for }|x|<b,\end{cases}
\end{aligned}
$$

where $2 \mathbb{Z}+1$ denotes the set of odd whole numbers.

Let us stress once more: the above result holds in the distributional sense only for test functions supported outside $x= \pm b$, i.e., for functions in that class there is

$$
\lim _{\lambda \rightarrow 0^{+}} \int \mathcal{E}_{a, \lambda}(x) \varphi(x) \mathrm{d} x=\int \mathcal{E}_{a}^{B}(x) \varphi(x) \mathrm{d} x .
$$

For functions not in this class our assumptions leading to the above result cease to hold. Nevertheless, we shall attempt some comparison with our global results. For that purpose let us denote

$$
\mathcal{E}^{B}(x)=-\frac{\sigma}{16 \pi^{2} x^{4}} \quad \text { for } x \neq 0 .
$$

Then (78) may be written as

$$
\mathcal{E}_{a}^{B}(x)=\mathcal{E}^{B}(x+b)+\mathcal{E}^{B}(x-b)+\mathcal{E}_{a, \text { int }}^{B}(x),
$$

where

$$
\mathcal{E}_{a, \text { int }}^{B}(x)= \begin{cases}+\frac{\sigma}{16 \pi^{2}(|x|+b)^{4}} & \text { for }|x|>b, \\ -\frac{\pi^{2}}{1440 a^{4}}-\sum_{n \in(2 \mathbb{Z}+1) \backslash\{+1,-1\}} \frac{\sigma}{16 \pi^{2}(n b-x)^{4}} & \text { for }|x|<b .\end{cases}
$$

It is easy to see that $\mathcal{E}_{a \text {,int }}^{B}(x)=a^{-4} F(x / a)$, where $F(y)$ is an absolutely bounded and integrable function. Thus for large separation of plates the energy outside the plates is concentrated in the first two terms in (80). Therefore (79) has the interpretation of the energy density produced around one single plate, while $\mathcal{E}_{a, \text { int }}^{B}(x)$ may be regarded as the energy density (locally outside the boundaries) of the interaction. When integrated over $x \in \mathbb{R}$ it gives

$$
\mathcal{E}_{a, \text { int }}^{B}=-\frac{\pi^{2}}{1440 a^{3}}
$$

for both Dirichlet and Neumann cases.

\section{Discussion}

The models considered in this article do not pretend to describe the details of the interaction of quantum field with macroscopic bodies in realistic way. Their merit comes from the fact that (i) they are consistent with the restrictions imposed by the general algebraic analysis of any quantum backreaction setting [1] while (ii) being simple enough to allow explicit calculations, and 
(iii) approximating sharp boundary conditions in a controllably localized (in physical space) way. The last point distinguishes them from the class of models constructed in [2] and allows the comparison of the results of the global and local analyses.

We summarize the results and lessons to be drawn from them.

(i) The models discussed in the present article are defined on the Weyl algebra of the free theory, and the interaction introduced by the 'nonlocal boundaries' does not lead out of the vacuum representation of the theory. The energy of the field (as defined by the free theory) is finite in the ground state (as defined by the interaction with 'boundaries') without any arbitrary 'renormalization'. The change of this 'Casimir energy' with the variation of the position of the boundaries determines the backreaction force.

(ii) The scaled interaction with the 'nonlocal boundaries' approaches in the scaling limit the sharp Dirichlet/Neumann conditions in the HilbertSchmidt norm sense for the resolvents. This implies norm convergence (or strong convergence) for any continuous, vanishing in infinity (resp. any bounded) function of the first-quantized hamiltonian. The nonlocality of the boundaries is under control and tends to zero in the limit.

(iii) The Casimir energy per area of the scaled models obeys the scaling law (56). Thus it is governed by the $a$-dependence of the energy given in Eqs. (57) and (58). The Casimir force per area is minus the derivative of those formulas. We have not discussed this point, but one can show that one can differentiate terms in these formulas one by one with $o\left(a^{-3}\right)$ going over to $o\left(a^{-4}\right)$. Thus one finds

$$
-\frac{\mathrm{d} \varepsilon_{a}^{\lambda}}{\mathrm{d} a}=\left\{\begin{array}{l}
-\frac{\pi^{2}}{480 a^{4}}+o\left(\frac{\lambda}{a^{4}}\right), \\
+\frac{1}{24 \pi M_{0} \lambda a^{3}}+\frac{I_{0}}{8 \pi^{2} M_{0}^{2} a^{4}}\left(\frac{3 \zeta(3)}{\pi^{2}}+1\right)-\frac{\pi^{2}}{480 a^{4}}+o\left(\frac{\lambda}{a^{4}}\right) .
\end{array}\right.
$$

One finds that in Dirichlet case the force has a well-defined limit, but in Neumann case depends on the model and diverges for sharp boundaries. This model-dependence occurs despite the fact that the models approximate in many respects the sharp boundaries very well (globally!). Neumann case models with $I_{0}=0$ (which are among those admitted by our assumptions - see Appendix C) have faster convergence property [see Eq. (35)] and for them the additional $a^{-4}$ term in the force vanishes.

(iv) Algebras of fields localized outside the interaction region (test functions with supports not intersecting with that region) admit free vacuum state, ground states of our models, as well as ground state of the sharp boundaries. Restricted to these algebras our ground states tend weakly to the sharp boundaries states in the scaling limit.

(v) The local energy density is unambiguously defined in our models in the whole physical space by point-splitting, with no ad hoc later renormalization. When restricted to regions not intersecting with the interaction 
area (which in scaling limit just means not intersecting with the boundaries) the local Casimir energy has a well defined limit given by a smooth function Eq. (78). This limit density has universal, model-independent form. Thus the 'bulk' contribution to the total Casimir energy has this universality and the model-dependent terms in Neumann case turn out to be squeezed in the limit inside the boundaries. The hope that these non-regular contributions may be removed in a model-independent way is therefore not justified. Further confirmation of our interpretation supply formulas and remarks ending the last section.

Local energy density has been discussed by various authors before [5-8], but usually with the use of some regularizations, often of not quite clear status. The results to be found in literature are not consistent. The Dirichlet case for the region between the plates is discussed in [7] and modulo some infinite renormalization agrees with ours (formula (2.32) in that reference). On the other hand the authors of [8] obtain a different result (by a rather indirect way of 'regularization' and removal of cut-off). We are not aware of a complete rigorous discussion resulting in our formulas (78)-(82). It is also worth noting that the density $\mathcal{E}_{a}^{B}(x)$ can be also obtained directly by the use of sharp boundary conditions Hamiltonian $h_{z a}^{B}$ instead of $h_{z a}$ in (77). This amounts to the use of the difference of (33) and (32) in the calculation. However, let us stress once more, this limit value of the density is correct only if smeared with a test function with support not touching the borders.

\section{Acknowledgements}

We are grateful to the referee for careful reading and constructive editorial remarks.

Open Access. This article is distributed under the terms of the Creative Commons Attribution Noncommercial License which permits any noncommercial use, distribution, and reproduction in any medium, provided the original author(s) and source are credited.

\section{Appendices}

If not stated otherwise we work here with the same assumptions as stated in (11) and (12).

\section{Appendix A. Integrals and Estimates}

We gather here a few technical, separate points used in the main text.

(i) We recall that $f$ is a smooth function supported in $\langle-R, R\rangle$ if, and only if, its Fourier transform $\widehat{f}$ is an entire analytic function satisfying the estimates 


$$
|\widehat{f}(u)| \leq \frac{\operatorname{const}(N) \mathrm{e}^{R|\mathfrak{I m} u|}}{(1+|u|)^{N}},
$$

for all $u \in \mathbb{C}$ and $N \in \mathbb{N}$.

Therefore $M_{p}$, which is the product of the Fourier transforms of $f(z)$ and of $\overline{f(-z)}=\overline{f(z)}$, extends to the analytic function $M_{u}$ on $\mathbb{C}$ satisfying similar estimates with $R$ replaced by $2 R$. The inverse transform $\check{M}$ of $M_{p}$ is smooth and supported in $\langle-2 R, 2 R\rangle$. Using this we find for $a>2 R$

$$
\int \cos (a p) M_{p} \mathrm{~d} p=\sqrt{\frac{\pi}{2}}[\check{M}(a)+\check{M}(-a)]=0 .
$$

As $M_{u}$ is even, $\frac{M_{p}-M_{k}}{p^{2}-k^{2}}$ also extends to an analytic function and with the use of estimates on $M_{u}$ one finds, by closing the contour of integration (as always for $a>2 R$ ), that

$$
\int \frac{\cos (a p)\left(M_{p}-M_{k}\right)}{p^{2}-k^{2}} \mathrm{~d} p=0 .
$$

(ii) In order to prove the estimate (30), we start with Dirichlet case, we note that (remember that $\widehat{f}^{\prime}(0)=0$, as $\widehat{f}$ is even)

$$
\left|\frac{\widehat{f}(\lambda p)-\widehat{f}(0)}{p^{2}}\right|=\left|\int_{0}^{\lambda} \widehat{f}^{\prime \prime}(p \xi)(\lambda-\xi) \mathrm{d} \xi\right| \leq \lambda \int_{0}^{\lambda}\left|\widehat{f}^{\prime \prime}(p \xi)\right| \mathrm{d} \xi .
$$

Moreover we have (norms of functions of $p$ as elements of $L^{2}$ )

$$
\begin{aligned}
& \left\|\int_{0}^{\lambda}\left|\widehat{f}^{\prime \prime}(p \xi)\right| \mathrm{d} \xi\right\|^{2} \leq \int_{\langle 0, \lambda\rangle^{2}}\left\|\widehat{f}^{\prime \prime}(p \xi)\right\|\left\|\widehat{f}^{\prime \prime}(p \tilde{\xi})\right\| \mathrm{d} \xi \mathrm{d} \tilde{\xi} \\
& \quad \leq\left\|\widehat{f}^{\prime \prime}\right\|^{2} \int_{\langle 0, \lambda\rangle^{2}}(\xi \tilde{\xi})^{-1 / 2} \mathrm{~d} \xi \mathrm{d} \tilde{\xi} \leq \operatorname{const} \lambda,
\end{aligned}
$$

where in the first step we have used the Schwartz inequality. Now because $\frac{p^{2}}{w^{2}-p^{2}}$ is bounded we get the estimate for Dirichlet. The same considerations show (31). The proof of Neumann case in (30) is almost the same but with the use of the formula

$$
\widehat{f}(\lambda p)-\widehat{f}(0)=p \int_{0}^{\lambda} \widehat{f}^{\prime}(p \xi) \mathrm{d} \xi .
$$

(iii) For the function $I_{k},(36)$, we first observe that for some $0<\epsilon_{0}<1$ there is

$$
I_{0}<2 \pi R M_{0}\left(1-\epsilon_{0}\right) .
$$


This follows from the identity

$$
4 \int_{0}^{\infty} \frac{\sin ^{2}(R p) M_{p}}{p^{2}} \mathrm{~d} p=2 \pi R M_{0}-I_{0},
$$

(used already in the normalization of the bound state, see $(43),(44)$ ), as the l.h.s. is strictly greater than zero. Further, we need the following estimates:

$$
\begin{array}{ll}
I_{k} \geq \frac{\operatorname{const}\left(k_{*}\right)}{k^{2}}, & k \geq k_{*}, \\
I_{k} \leq \frac{\mathrm{const}}{(1+k)^{2}}, & k \geq 0,
\end{array}
$$

with arbitrary $k_{*}>0$. We write $I_{k}$ as a principal value distribution calculated on test function $M$. In position space, using evenness of $f$, we have

$$
I_{k}=\sqrt{2 \pi} \int_{0}^{\infty} x \check{M}(x) \frac{\sin (k x)}{k x} \mathrm{~d} x .
$$

Integrating once by parts we get

$$
I_{k}=\frac{\sqrt{2 \pi}}{k^{2}}\left[\check{M}(0)+\int_{0}^{\infty} \check{M}^{\prime}(x) \cos (k x) \mathrm{d} x\right] .
$$

The estimation from above is now trivial, whereas for the estimation from below we use the Riemann-Lebesgue lemma, the assumption that $I_{k}>0$ for $k \neq 0$ [see (37)] and continuity of $I_{k}$. Expanding further in powers of $1 / k$ the integral in (89) we find moreover

$$
\left|\partial_{k}^{n} I_{k}\right| \leq \frac{\text { const }}{(1+k)^{n+2}}
$$

(iv) The $s_{k}$ function, defined in the end of Sect. 4, is smooth for $k \geq 0$ and satisfies

$$
\left|\partial_{k}^{n} s_{k}\right| \leq \operatorname{const}(n)(1+k)^{-(n+\sigma)} .
$$

To show this, we note first that $\left|M_{k}-i N_{k}\right|^{-1}$ is bounded in a neighborhood of $k=0$ as $M_{0}>0$. Outside this neighborhood we have $\left|M_{k}-i N_{k}\right|^{-1} \leq$ $\left|N_{k}\right|^{-1} \leq \operatorname{const}(1+k)^{-\sigma}$ due to (38) and the first bound in (87). On the other hand, due to (90) there is $\left|\partial_{k}^{n} N_{k}\right| \leq \operatorname{const}(1+k)^{-(n+1)}$ for $n \geq 1$ except for $n=1$ in the Dirichlet case, when this is replaced by $\left|\partial_{k} N_{k}\right| \leq$ const. As $M_{k}$ is Schwartz, this ends the proof.

It now follows that $q_{k}=s_{k} M_{k}$ and all its derivatives are bounded by Schwartz functions.

(v) For both (D and $\mathrm{N})$ cases and $k \geq 0$ we note the bound

$$
\frac{1}{\left|1-\left(q_{k} \mathrm{e}^{i a k}\right)^{2}\right|} \leq \operatorname{const}(a) \frac{1+k}{k} \text {. }
$$

At $k=0$ there is $1-\left(q_{0}\right)^{2}=0$ but the first derivative of the denominator at zero does not vanish (in Neumann case use (86)), which is sufficient for (92) 
in a neighborhood of zero. Outside that neighborhood, mainly due to (87), it follows that

$$
\frac{1}{\left|1-\left(q_{k} \mathrm{e}^{i a k}\right)^{2}\right|} \leq \frac{1}{1-\left|q_{k}\right|^{2}}=\frac{M_{k}^{2}+N_{k}^{2}}{N_{k}^{2}} \leq \mathrm{const},
$$

which ends the proof. We also note that for $k \geq 0$ we have

$$
\frac{1}{1-\left|q_{k}\right|^{2}} \leq \operatorname{const} \frac{1+k^{m}}{k^{m}}, \quad \begin{cases}m=2, & (\mathrm{D}) \\ m \geq 2, & (\mathrm{~N})\end{cases}
$$

where the Neumann case depends on the behavior of $I_{k}$ at zero $(m=2$ for $I_{0} \neq 0$ and $m=2+4 r$ when $I_{k} \underset{k \rightarrow 0}{\simeq} k^{2 r}, r \geq 1$ as $I_{k}$ is even).

(vi) Finally, we note the following identity using a known integral representation of the Hurwitz zeta function: for $\alpha>0$ there is

$$
\frac{1}{6} \int_{0}^{\infty} \frac{r^{3} \mathrm{e}^{-\alpha r}}{1-\mathrm{e}^{-2 a r}} \mathrm{~d} r=\frac{1}{(2 a)^{4}} \zeta\left(4, \frac{\alpha}{2 a}\right)=\sum_{n=0}^{\infty}(\alpha+2 a n)^{-4},
$$

which is needed for the calculation of (78).

\section{Appendix B. Operations (i)-(iii) from Section 7}

In this appendix we prove the admissibility of the three operations (i)-(iii) performed in Sect. 7.1 for the Dirichlet case and mentioned in Sect. 7.2 for the Neumann case. The key tool for this is the following simple lemma.

Let $c_{n}$ for $n \in \mathscr{N} \subseteq \mathbb{N}$ be complex measurable functions on $D \subseteq \mathbb{R}$. If $\sum_{n \in \mathscr{N}}\left|c_{n}(k)\right|$ is integrable on $D$ then

$$
\lim _{a \rightarrow \infty} \sum_{n \in \mathscr{N}} \int_{D} c_{n}(k) \mathrm{e}^{i n a k} \mathrm{~d} k=0 .
$$

The proof is straightforward and uses the Lebesgue dominated convergence theorem and the Riemann-Lebesgue lemma. Throughout this appendix $\mathscr{S}_{k}$ denotes some Schwartz function in $k$ variable (each time it may be a different function). All we have to do is to check if the assumption of the mentioned lemma is fulfilled for the appropriate expressions.

\section{B.1. Dirichlet Case}

Let us first consider the following part of the expression (64)

$$
\frac{1}{a^{3}} \sum_{n \in 2 \mathbb{N}} \frac{i}{n^{3}} \int_{\mathbb{R}_{+}^{2}} M_{p} \partial_{k}^{3}\left(\chi(k, p) s_{k} q_{k}^{n}\right) \mathrm{e}^{i n a k} \mathrm{~d} k \mathrm{~d} p ;
$$

we shall need its real part multiplied by $-1 / 3 \pi^{3}$ in (64). 
(i) To justify the validity of operation (i) of Sect. 7.1 it is sufficient to check whether the sequence of functions

$$
c_{n}(k)=n^{-3} \int_{0}^{\infty} M_{p}\left[\partial_{k}^{3}\left(s_{k} q_{k}^{n} \chi(k, p)\right)-s_{k} q_{k}^{n} \partial_{k}^{3} \chi(k, p)\right] \mathrm{d} p
$$

satisfies the assumption of lemma (95). For this we note that using properties of $s_{k}$ and $q_{k}$ described in Appendix A (iv) we have

$$
\begin{aligned}
& n^{-3}\left|\partial_{k}^{3}\left(s_{k} q_{k}^{n} \chi(k, p)\right)-s_{k} q_{k}^{n} \partial_{k}^{3} \chi(k, p)\right| \\
& \leq \mathscr{S}_{k}\left(n^{-3}\left|q_{k}\right|^{n-1}+n^{-2}\left|q_{k}\right|^{n-1}\right) \sum_{j=0}^{2}\left|\partial_{k}^{j} \chi(k, p)\right| \\
& \quad+\mathscr{S}_{k} n^{-2}(n-1)\left|q_{k}\right|^{n-2} \sum_{j=0}^{1}\left|\partial_{k}^{j} \chi(k, p)\right| \\
& \quad+\mathscr{S}_{k} n^{-2}(n-1)(n-2)\left|q_{k}\right|^{n-3}|\chi(k, p)| .
\end{aligned}
$$

A simple calculation yields

$$
\left|\partial_{k}^{j} \chi(k, p)\right| \leq \mathrm{const} \frac{k^{2-j}}{k+p}, \quad j=0,1,2,
$$

and therefore

$$
\int_{0}^{\infty} M_{p}\left|\partial_{k}^{j} \chi(k, p)\right| \mathrm{d} p \leq \mathrm{const} k^{2-j} \log \left(1+k^{-1}\right), \quad j=0,1,2 .
$$

Using this and remembering that $\left|q_{k}\right| \leq 1$ we obtain

$$
\left|c_{n}(k)\right| \leq \mathscr{S}_{k} \log \left(1+k^{-1}\right)\left(n^{-3}+n^{-2}+n^{-1} k\left|q_{k}\right|^{n-2}+k^{2}\left|q_{k}\right|^{n-3}\right),
$$

where the third and the fourth terms inside the parentheses appear for $n \geq 2$ and $n \geq 3$ respectively. The summation gives

$$
\sum_{n \in 2 \mathbb{N}}\left|c_{n}(k)\right| \leq \mathscr{S}_{k} \log \left(1+k^{-1}\right)\left[1+k \log \left(\frac{1}{1-\left|q_{k}\right|^{2}}\right)+\frac{k^{2}}{1-\left|q_{k}\right|^{2}}\right] \text {. }
$$

The estimate (93) shows that the integral of the r.h.s. over $\mathbb{R}_{+}$is finite, thus the assumption of lemma (95) is satisfied.

(ii) For the operation (ii) the assumption of the lemma is fulfilled because

$$
\left|M_{p} s_{k} q_{k}^{n} \partial_{k}^{3} \chi(k, p)\right| \leq \frac{6 p^{2}(k+3 p)}{(k+p)^{5}} M_{p} \mathscr{S}_{k} \in L^{1}\left(\mathbb{R}_{+}^{2} \backslash \Omega\right),
$$

with $\Omega$ as defined in Sect. 7.1.

(iii) The operation (iii) is admissible because

$$
\left|M_{p} s_{k} q_{k}^{n}-M_{0} s_{0} q_{0}^{n}\right| \leq \text { const } p+\operatorname{const}(1+n) k+\operatorname{const}(1+n) p k
$$

and $k \partial_{k}^{3} \chi(k, p), p \partial_{k}^{3} \chi(k, p)$ are both integrable on $\Omega$, so the lemma holds also in this case. This ends the proof for the terms (96). 
For the expression with the sum over odd natural numbers and with $\cos (a p)$ we use the following modification of the lemma. Let $c_{n}$ for $n \in \mathscr{N} \subseteq \mathbb{N}$ be complex measurable functions on $D \subseteq \mathbb{R}^{2}$. If $\sum_{n \in \mathscr{N}}\left|c_{n}(k, p)\right|$ is integrable on $D$ then

$$
\lim _{a \rightarrow \infty} \sum_{n \in \mathscr{N}} \int_{D} c_{n}(k, p) \mathrm{e}^{i n a k} \mathrm{e}^{ \pm i a p} \mathrm{~d} k \mathrm{~d} p=0 .
$$

Now, almost the same considerations as before show the admissibility of operations (i)-(iii) for this part of energy.

\section{B.2. Neumann Case}

First we note that for the terms which we consider here, the limit over $\epsilon$ can be easily performed. This follows from the estimations below. Therefore we use the same lemma as for Dirichlet case, i.e., (95) and (102), but we recall that we now replace $q_{k} \mathrm{e}^{i a k}=\tilde{q}_{k} \mathrm{e}^{i \tilde{a} k}$, as mentioned at the beginning of Sect. 7.2. With this modification the estimation (97) for the terms in the sum (96) is still valid, but the bounds (98) change: $k^{2-j}$ is replaced by $p^{2-j}$. In consequence there is no $k^{2-j}$ factor in front of $\log$ in (99) and no $k, k^{2}$ factors in (100) and (101). With this modification the sum of $n^{-3}, n^{-2}$ and $n^{-1}$ terms in (100) is still sufficiently well bounded, but the sum of $n^{0}$ terms is to singular (no $k^{2}$ in the last term in (101)). Therefore these terms need a more detailed treatment. In fact, they can be estimated by const $\left|s_{k}\right|\left|\tilde{q}_{k}\right|^{n-3}\left|\tilde{q}_{k}^{\prime}\right|^{3} \log \left(1+k^{-1}\right)$ (prime denotes here the derivative with respect to $k$ ) and their sum over $n$ is bounded by

$$
\text { const } \frac{\left|s_{k}\right|\left|\tilde{q}_{k}^{\prime}\right|^{3}}{1-\left|\tilde{q}_{k}\right|^{2}} \log \left(1+k^{-1}\right) .
$$

This function is indeed in $L^{1}\left(\mathbb{R}_{+}\right)$, since outside the neighborhood of zero, using (93), we have $\frac{\left|s_{k}\right|\left|\tilde{q}_{k}^{\prime}\right|^{3}}{1-\left|\tilde{q}_{k}\right|^{2}} \leq \mathscr{S}_{k}$, whereas in the neighborhood we have $\tilde{q}_{0}^{\prime}=0$ which is enough for $I_{0} \neq 0$ case [see (93) and the comment after it] and if $I_{k} \underset{k \rightarrow 0}{\simeq} k^{2 r}, r \geq 1$ ( $I_{k}$ is even), then $\frac{\left|\tilde{q}_{k}^{\prime}\right|^{3}}{1-\left|\tilde{q}_{k}\right|^{2}} \underset{k \rightarrow 0}{\simeq} k^{2 r-2}$. The discussion of admissibility of operations (ii) and (iii) goes in the same way as for Dirichlet case (estimates hold also for Neumann case, with $\tilde{q}_{k}$ instead of $q_{k}$ ). The analysis of the expression with the sum over odd natural numbers and with $\cos (a p)$ is also analogous to the Dirichlet case.

\section{Appendix C. Comments on the Assumed Class of Models}

The models discussed in this paper are based on functions $f$ subject to the conditions formulated in (11), (12), (37) and (38). In this appendix we exhibit a class of functions conforming to them. It is sufficient to satisfy (11) and (37) as the other two are then achieved by simple rescaling of the function by a constant factor. 
First, we note that each even function with the assumed support which in addition is real, non-negative and monotonically (weakly) decreasing for positive arguments satisfies the demands. Indeed, for each such function the last condition in (11) is fulfilled. Moreover, it is easy to see that then $\check{M}(x)$ (being the convolution of the function with itself) is also even, positive, compactly supported and decreasing for $x>0$. Thus from (89), since for $k \neq 0$ there is

$$
\left|\int_{0}^{\infty} \check{M}^{\prime}(x) \cos (k x) \mathrm{d} x\right|<-\int_{0}^{\infty} \check{M}^{\prime}(x) \mathrm{d} x=\check{M}(0),
$$

we have $I_{k}>0$ for $k \neq 0$, which ends the proof of (37).

For each function in the class defined in the previous paragraph there is $I_{0}>0$, which is due to the positivity of $\check{M}(x)$ [see (88)]. We now extend the class to include also functions for which $I_{0}=0$ (see Discussion). Let $f$ be a function in the class of the last paragraph and define a new function

$$
f^{r}(z)=f(z)-\mu(f(z-r)+f(z+r)),
$$

where $\mu>0$ and $r>R>0$. One finds

$$
M_{p}^{r}=\left|\widehat{f}^{r}(p)\right|^{2}=(1-2 \mu \cos (r p))^{2} M_{p}
$$

Using this and taking into account (85) one has

$$
I_{k}^{r}=\int \frac{M_{k}^{r}-M_{p}^{r}}{p^{2}-k^{2}} \mathrm{~d} p=\left(1+2 \mu^{2}\right) I_{k}-4 \pi \mu \frac{\sin (r k)}{k}(1-\mu \cos (r k)) M_{k} .
$$

We impose the condition $I_{0}^{r}=0$, which is a quadratic equation for $\mu$ :

$$
2\left(I_{0}+2 \pi r M_{0}\right) \mu^{2}-4 \pi r M_{0} \mu+I_{0}=0 .
$$

For sufficiently large $r$ the equation has two roots, and we take the smaller one, which is less then $1 / 2$ and for large $r$ tends to zero. Then

$$
I_{k}^{r}=4 \pi r \mu(1-\mu) M_{0} \frac{I_{k}}{I_{0}}[1-\eta(r k) \xi(k)],
$$

where

$$
\eta(r k)=\frac{\sin (r k)}{r k} \frac{1-\mu \cos (r k)}{1-\mu}, \quad \xi(k)=\frac{I_{0} M_{k}}{M_{0} I_{k}} .
$$

It is an exercise in function analysis to show that for sufficiently small $\mu$ there is $\eta(u)<1$ for all $u \neq 0$. Below we show that $f$ may be chosen such that also $\xi(k)<1$ for $k \neq 0$, and then $I_{k}^{r}>0$ for $k>0$, which is the condition (37). To reduce the size of the support of $f^{r}$ one can use the scaling defined in Sect. 3.

Consider now the non-negative and even function $\xi(k)$ for positive arguments. Suppose that $\xi^{\prime \prime}(0)<0$ (see below). Then there is $k_{0}>0$ such that for $k \in\left(0, k_{0}\right)$ it is $\xi(k)<1$. For $k \geq k_{0}$, using $(87)$, we have $\xi(k) \leq$ const $\equiv \xi_{\text {max }}$. We now need to improve the estimation of $\eta$ for $k \geq k_{0}$. It is easy to see that if $r$ has been chosen large enough then $\eta(r k)<\frac{1}{\xi_{\max }}$ for $k \geq k_{0}$. 
Finally, we have to choose $f$ so as to satisfy $\xi^{\prime \prime}(0)<0$. Let $f$ be defined as $f(z)=f_{0}=$ const on $\langle-R, R\rangle$ and zero outside. Straightforward calculation gives

$$
\begin{gathered}
M_{k}=\frac{2\left|f_{0}\right|^{2}}{\pi} R^{2} \frac{\sin ^{2}(R k)}{(R k)^{2}}, \quad I_{k}=8\left|f_{0}\right|^{2} R^{3} \frac{2 R k-\sin (2 R k)}{(2 R k)^{3}}, \\
\xi^{\prime \prime}(0)=-\frac{4}{15} R^{2}<0 .
\end{gathered}
$$

The function used here needs 'rounding the corners' to be in the class of the second paragraph. But this may be made by a small local variation, and both $M_{k}$ and $I_{k}$ and their derivatives depend continuously on such small variations of $f$ [for $I_{k}$ see (88)], which is sufficient to conclude the proof.

\section{References}

[1] Herdegen, A.: Ann. Henri Poincaré 6, 669-707 (2005)

[2] Herdegen, A.: Ann. Henri Poincaré 7, 253-301 (2006)

[3] Jaffe, A., Williamson, L.R.: Ann. Phys. 282, 432 (2000)

[4] Fosco, C.D., Losada, E.: Phys. Lett. B 675, 252 (2009)

[5] Lütken, C.A., Ravndal, F.: Phys. Rev. A 31, 2082 (1985)

[6] Sopova, V., Ford, L.H.: Phys. Rev. D 66, 045026 (2002)

[7] Milton, K.A.: Phys. Rev. D 68, 065020 (2003)

[8] Kawakami, N.A., Nemes, M.C., Wreszinski, W.F.: J. Math. Phys. 48, 102302 (2007)

Andrzej Herdegen and Mariusz Stopa

Institute of Physics

Jagiellonian University

Reymonta 4

30-059 Cracow

Poland

e-mail: herdegen@th.if.uj.edu.pl

e-mail: stopa@th.if.uj.edu.pl

Communicated by Klaus Fredenhagen

Received: April 12, 2010

Accepted: July 6, 2010 